\title{
Dual-color decompositions at one-loop level in Yang-Mills theory
}

\author{
Yi-Jian Du, ${ }^{a, b}$ Bo Feng ${ }^{c, d}$ and Chih-Hao Fu ${ }^{e}$ \\ ${ }^{a}$ Department of Physics and Center for Field Theory and Particle Physics, Fudan University, \\ Shanghai 200433, P.R. China \\ ${ }^{b}$ Department of Astronomy and Theoretical Physics, Lund University, \\ SE-22362 Lund, Sweden \\ ${ }^{c}$ Zhejiang Institute of Modern Physics, Zhejiang University, \\ 38 Zheda Road Hangzhou, 310027 P.R. China \\ ${ }^{d}$ Center of Mathematical Science, Zhejiang University, \\ 38 Zheda Road Hangzhou, 310027 P.R. China \\ ${ }^{e}$ Department of Electrophysics, National Chiao Tung University, \\ 1001 University Road, Hsinchu, Taiwan, R.O.C. \\ E-mail: yjdu@fudan.edu.cn, b.feng@cms.zju.edu.cn, zhihaofu@nctu.edu.tw
}

ABSTRACT: In this work we extend two dual color decompositions of Yang-Mills amplitude to one-loop level. Starting from double-copy expression we translate the Yang-Mills integrand into the dual Del Duca-Dixon-Maltoni formulation and subsequently to the dual color-ordered formula. The dual trace factors are obtained after simultaneously solving the one-loop Kleiss-Kuijf relations, the reflection relation, and the relation that expresses the kinematic factors in the dual Del Duca-Dixon-Maltoni form.

KEywords: Scattering Amplitudes, Gauge Symmetry

ARXiv EPRINT: 1402.6805 


\section{Contents}

1 Introduction 1

2 Dual DDM-form $\quad 6$

2.1 Two-point example 6

$\begin{array}{lll}2.2 & \text { Three-point example } & 7\end{array}$

2.3 Four-point example 8

3 Dual trace-form $\quad 11$

3.1 General structure of the defining conditions 11

$\begin{array}{lll}3.2 & \text { Four-point dual traces } & 15\end{array}$

$\begin{array}{lll}3.3 & \text { Five-point case } & 16\end{array}$

$\begin{array}{lll}3.4 & \text { Six-point example } & 16\end{array}$

$\begin{array}{lll}4 & \text { An alternative approach } & 20\end{array}$

5 Conclusion $\quad 22$

\section{Introduction}

One of the significant recent progresses in the study of scattering amplitudes is the discovery of color-kinematic duality by Bern, Carrasco and Johansson (BCJ) [1]. It was conjectured that a generic $L$-loop Yang-Mills amplitude can be expressed as a double-copy formula,

$$
\mathcal{A}_{\mathrm{tot}}^{L}=i^{L} g^{m-2+2 L} \sum_{D_{i}} \int \prod_{j=1}^{l} \frac{d^{D} l_{j}}{(2 \pi)^{D}} \frac{1}{S_{i}} \frac{n_{i}(l) c_{i}}{\prod_{k} P_{k i}(l)},
$$

For the purpose of discussion, in this paper we shall call the above expression as BCJ form so that it makes better distinction from the rest of the formulations. In equation (1.1) the summation is taken over all possible cubic Feynman-like diagrams and $S_{i}$ is a symmetry factor. The color factor $c_{i}$ in this formula is consisted of group structure constants $f^{a b c}$ whereas $n_{i}(l)$ is the kinematic factor satisfying the following properties: Whenever two color factors $c_{i}, c_{j}$ are related by antisymmetry or three color factors $c_{i}, c_{j}, c_{k}$ by Jacobi identity, so are the corresponding kinematic factors $n$.

$$
\begin{aligned}
& \text { antisymmetry : } \quad c_{i}=-c_{j} \quad \Rightarrow \quad n_{i}=-n_{j} \\
& \text { Jacobi - like identity : } \quad c_{i}+c_{j}+c_{k}=0 \quad \Rightarrow n_{i}+n_{j}+n_{k}=0 .
\end{aligned}
$$

At tree-level the existence of BCJ form was proven from string theory in [2, 3], and in particular from twistor string theory [4-7]. A proof from purely field theory perspective 
can be found in [8-10], while a wide range of relevant studies can be found in the following list of papers [11-19] (see also [20] for a nice review). At loop-level the BCJ form remains a conjecture, however a considerable amount of support can be found in the literature [21-31].

The apparent equal-footing treatment of the color and kinematic factors of (1.1) introduces a very interesting perspective to the understanding of the structure of Yang-Mills amplitudes. To see its implication more clearly, let us review a few results at tree-level.

Tree-level formulations. At tree-level, we can write Yang-Mills amplitudes in the following three color decomposition forms $[32,33]$ :

$$
\begin{aligned}
\text { Double-copy (or BCJ) form : } & \mathcal{A}_{\mathrm{tot}}=\sum_{i} \frac{c_{i} n_{i}}{D_{i}} \\
\text { Trace form : } & \mathcal{A}_{\mathrm{tot}}=\sum_{\sigma \in S_{n-1}} \operatorname{Tr}\left(T^{\sigma_{1}} \ldots T^{\sigma_{n}}\right) A(\sigma) \\
\text { DDM form : } & \mathcal{A}_{\mathrm{tot}}=\sum_{\sigma \in S_{n-2}} c_{1|\sigma(2, \ldots, n-1)| n} A(1, \sigma, n)
\end{aligned}
$$

Here Roman letters $A$ represent color ordered amplitudes, $T^{a}$ are generators of $\mathrm{U}(N)$ in fundamental representation, and $c_{i}, c_{1|\sigma(2, \ldots, n-1)| n}$ represent strings of structure constants $f^{a b c}$

$$
c_{1|\sigma(2, \ldots, n-1)| n}=f^{1 \sigma_{2} x_{1}} f^{x_{1} \sigma_{3} x_{2}} \ldots f^{x_{n-3} \sigma_{n-1} n} .
$$

Among these three forms, the relation between the trace form (1.4) and the Del DucaDixon-Maltoni half ladder formulation (1.5) (abbreviated as DDM form in this paper) has been well understood using the following two properties of the Lie algebra of $\mathrm{U}(N)$ gauge group (see ref. [33])

$$
\begin{array}{ll}
\text { Property One : } & \left(f^{a}\right)_{i j}=f^{a i j}=\operatorname{Tr}\left(T^{a}\left[T^{i}, T^{j}\right]\right), \\
\text { Property Two : } & \sum_{a} \operatorname{Tr}\left(X T^{a}\right) \operatorname{Tr}\left(T^{a} Y\right)=\operatorname{Tr}(X Y)
\end{array}
$$

On the other hand the existence of BCJ form (1.3) is far from trivial and has sparked the intense study introduced at the beginning of this section. For special helicity configurations, it was shown that the kinematic numerators correspond to area-preserving diffeomorphism algebra $[16,17]$. Using this idea, an explicit construction of the BCJ numerators $n_{i}$ was given in [18], thereby providing a support to an algebra-manifest formulation. Similarly, Mafra, Schlotterer and Stieberger have given an explicit construction in [15] using Berkovits' pure spinor formalism. Finally, using the twistor string theory, Cachazo, He and Yuan gave an algorithm for $n_{i}$ using solutions to the scattering equations [7].

Although it is seemingly quite difficult to derive BCJ form from the trace or DDM form, it is not as much hard to derive the trace and the DDM form from the opposite direction. Explicitly, using Jacobi relations, one can construct a basis of all the color factors $c_{i}$, which are simply the factors given in (1.6). Expanding color factors into this basis, $c_{i}=\sum_{\sigma} \alpha_{\sigma} c_{1|\sigma| n}$, and collecting terms rewrites the original BCJ form (1.3) as

$$
\mathcal{A}_{\mathrm{tot}}=\sum_{\sigma \in S_{n-2}} c_{1|\sigma(2, \ldots, n-1)| n} \widehat{A}(1, \sigma, n)
$$


A crucial question that we need to answer before claiming equation (1.9) to be the same as the desired DDM form (1.5) is that whether the coefficients $\widehat{A}(1, \sigma, n)$ obtained here through collecting terms are the same as the partial amplitudes $A(1, \sigma, n)$ given in (1.5). This identification can be argued either from the fact that basis color factors $c_{1|\sigma(2, \ldots, n-1)| n}$ by definition must be independent to each other and that both $\widehat{A}$ and $A$ are minimum gauge invariant objects, or by using the KLT relation [34] as in [35].

In viewing of the fact that in BCJ form $n_{i}$ acquires the same status as $c_{i}$, it is natural to exchange the roles between $c_{i}$ and $n_{i}$ and consider the following two dual formulations

$$
\begin{aligned}
\text { Dual trace form : } & \mathcal{A}_{\text {tot }}=\sum_{\sigma \in S_{n-1}} \tau_{\sigma_{1} \ldots \sigma_{n}} \widetilde{A}(\sigma) \\
\text { Dual DDM form : } & \mathcal{A}_{\text {tot }}=\sum_{\sigma \in S_{n-2}} n_{1|\sigma(2, \ldots, n-1)| n} \widetilde{A}(1, \sigma, n)
\end{aligned}
$$

where $\widetilde{A}$ is the color-ordered scalar amplitude with $f^{a b c}$ as its cubic coupling constant (see refs. [35, 36]) and $\tau$ is required to be cyclic invariant. The idea of a dual DDM-form first appeared in the literature in [37]. Using the Jacobi identity we can expand a generic numerator into a set of basis numerators, $n_{i}=\sum_{\sigma} n_{1|\sigma| n}$, through a parallel operation to what was performed on the color factor $c_{i}$. Plugging these relations back to the BCJ form (1.3) and collecting terms leads to the form given in (1.11). Note however, it is far from obvious that the $\widetilde{A}$ obtained this way reproduces the same color-ordered scalar amplitude as was claimed by (1.11), which is defined by cubic Feynman rules with $f^{a b c}$ as its coupling. To make this identification, an idea is to perform the analogous manipulation of [35] on KLT relation, and this requires the off-shell BCJ gauge theory amplitude relation presented in [18]. Based on the existence of a dual DDM form, the dual trace form was conjectured by Bern and Dennen in [38] with explicit constructions given for the first few lower-point amplitudes. In addition two constructions of the dual trace form was discussed in [40] and [41].

One-loop level. Having reviewed the tree-level case, let us move on to the one-loop case. At one-loop level we have the following three formulations of total amplitude:

$$
\begin{aligned}
\text { BCJ form : } \mathcal{A}_{\text {tot }}^{1-\text { loop }}= & i g^{n} \sum_{\text {diagrams } \Gamma^{i}} \int \prod \frac{d^{D} l}{(2 \pi)^{D}} \frac{1}{S_{i}} \frac{n_{i}(l) c_{i}}{\prod_{k} P_{k i}(l)} \\
\text { Trace form : } \mathcal{A}_{\text {tot }}^{1-\text { loop }}= & N_{c} \sum_{S_{n} / Z_{n}} \operatorname{Tr}\left(T^{\sigma_{1}} \ldots T^{\sigma_{n}}\right) A_{n ; 0}\left(\sigma_{1}, \ldots, \sigma_{n}\right) \\
& +\sum_{m=1}^{\lfloor n / 2\rfloor} \sum_{\sigma \in S_{n} / S_{n ; m}} \operatorname{Tr}\left(T^{\sigma_{1}} \ldots T^{\sigma_{n-m}}\right) \operatorname{Tr}\left(T^{\sigma_{n-m+1}} \ldots T^{\sigma_{n}}\right) \\
& \times A_{n-m ; m}\left(\sigma_{1}, \ldots, \sigma_{n-m} ; \sigma_{n-m+1}, \ldots, \sigma_{n}\right) \\
\text { DDM form : } \mathcal{A}_{\text {tot }}^{1-\text { loop }}= & \sum_{\sigma \in S_{n-1} / R} f^{x_{n} \sigma_{1} x_{1}} f^{x_{1} \sigma_{2} x_{2}} \ldots f^{x_{n-1} \sigma_{n} x_{n}} A_{n, 0}\left(\sigma_{1}, \ldots, \sigma_{n}\right)
\end{aligned}
$$

In (1.13), $Z_{n}$ denotes cyclic permutations, $S_{n ; m}$ is the subset of $S_{n}$ that leaves the doubletrace structure invariant and $\lfloor n / 2\rfloor$ is the greatest integer less than or equal to $n / 2$. The $R$ 
in equation (1.14) denotes reflection. At the moment of writing, the last two formulations, i.e., (1.13) and (1.14), are proven while the BCJ form (1.12) at generic $n$-point at one-loop level remains a conjecture.

At one-loop level the trace form consists of both single and double trace parts [42], where the partial amplitude $A_{n-m ; m}\left(\sigma_{1}, \ldots, \sigma_{n-m} ; \sigma_{n-m+1}, \ldots, \sigma_{n}\right)$ associated to the double trace part can be obtained by linear combination of those to the single trace part $A_{n ; 0}\left(\sigma_{1}, \ldots, \sigma_{n}\right)$. In other words $A_{n-m ; m}\left(\sigma_{1}, \ldots, \sigma_{n-m} ; \sigma_{n-m+1}, \ldots, \sigma_{n}\right)$ does not really bring extra information to the one-loop amplitude. The DDM form given in [33] on the other hand consists of summation over noncylic permutations up to reflections $R$ : $(12 \ldots n)=(n \ldots 21)$. Here the $A_{n ; 0}\left(\sigma_{1}, \ldots, \sigma_{n}\right)$ in (1.14) is nothing but the same single trace partial amplitude appeared in (1.13). In fact it was demonstrated in [33] that it is straightforward to derive the trace form starting from DDM form, and the relation between single and double trace partial amplitudes appears automatically as a by-product.

Assuming the existence of (1.12), going from BCJ form to the trace form (1.13) or to the DDM form (1.14) is relatively easy. As shown by Del Duca, Dixon and Maltoni in [33], for the one-loop color factors, annuli of structure constants of the form

$$
c^{1-\operatorname{loop}}\left(\sigma_{1} \ldots \sigma_{n}\right) \equiv f^{x_{n} \sigma_{1} x_{1}} f^{x_{1} \sigma_{2} x_{2}} \ldots f^{x_{n-1} \sigma_{n} x_{n}}
$$

serve as a basis. Using it we can expand any $c_{i}$ in BCJ form and collect terms carrying the same color factor $c^{1-\operatorname{loop}}\left(\sigma_{1} \ldots \sigma_{n}\right)$. Suppose if we call the collection of coefficients as $\widehat{A}_{n, 0}\left(\sigma_{1}, \ldots, \sigma_{n}\right)$, again the crucial problem is whether it equals the amplitude $A_{n, 0}\left(\sigma_{1}, \ldots, \sigma_{n}\right)$ defined in (1.14)? The identification is again easy because by definition the color basis $c^{1-\text { loop }}\left(\sigma_{1} \ldots \sigma_{n}\right)$ consists of independent elements, and that $\widehat{A}$ and $A$ are gauge invariant objects.

The above discussions are parallel to those given at tree-level. Considering the duality between $n_{i}$ and $c_{i}$ in (1.12), it is natural to investigate the dual form [38], where the interest of this paper lies. ${ }^{1}$ Unlike color numerators, the $n_{i}$ depends on the loop momentum in general, so the dual formulations at one-loop are expected to take the following forms: ${ }^{2}$

$$
\mathcal{A}_{1-\text { loop }}=i g^{n} \int \frac{d^{D} l}{(2 \pi)^{D}} \sum_{\sigma \in S_{n-1}} n_{1, \sigma}^{\mathrm{DDM}}(l) \widetilde{I}(1, \sigma) .
$$

for the dual DDM-form, and

$$
\begin{aligned}
\mathcal{A}_{1-\text { loop }}= & i g^{n} \int \frac{d^{D} l}{(2 \pi)^{D}} \sum_{m=0}^{\lfloor n / 2\rfloor} \sum_{\sigma \in S_{n} / S_{n ; m}} \tau_{\left\{\sigma_{1}, \ldots, \sigma_{n-m}\right\},\left\{\sigma_{n-m+1}, \ldots, \sigma_{n}\right\}}(l) \\
& \times \widetilde{I}\left(\left\{\sigma_{1}, \ldots, \sigma_{n-m}\right\} ;\left\{\sigma_{n-m+1}, \ldots, \sigma_{n}\right\}\right),
\end{aligned}
$$

\footnotetext{
${ }^{1}$ In supergravity theory, a 'dual DDM-form'(where the full amplitude is decomposed according to one of the two copies of BCJ numerators) decomposition at one-loop level of supergravity has already been suggested in [39] and the dual DDM-form in Yang-Mills theory has the similar form. Nevertheless, in this work, we would like to give a general discussion on dual DDM-form at one-loop level in Yang-Mills theory in the introduction and some explicit examples in section 2, because the dual DDM-form is crucial for the construction of dual trace-form.

${ }^{2}$ One may notice that in one-loop DDM form (1.14), reflection has been modded out, in the dual DDMform, we just leave the reflection symmetry and only consider it when we discuss on the dual trace-form.
} 
for the dual trace-form. ${ }^{3}$ Therefore inevitably, the duality needs to be considered at integrand level. In the light of preceding success in deriving dual DDM forms from BCJ forms, it is natural to wonder if similar idea works at one-loop, and we see that collecting coefficients in a suitable basis of $n_{i}$ indeed returns the dual DDM form, albeit a few complexities introduced by the loop momentum dependence, particularly in identifying what integrand these collected coefficients correspond to.

More explicitly, starting from BCJ form one decomposes numerators with respect to any given loop momentum alignment convention, $n_{i}(l)=\sum_{\alpha} \kappa_{i \alpha} n_{\alpha}\left(l^{\prime}\right)$, and the integrand is given by

$$
\sum_{\alpha} n_{\alpha}(l)\left(\sum_{i \text { :diagrams }} \kappa_{i \alpha} \frac{c_{i}}{D_{i}}\right)
$$

up to terms that vanish after the integration. The factors inside the bracket can be identified as colored scalar integrand with respect to certain loop momentum alignment convention. ${ }^{4}$ In section 2 , we will demonstrate how this can be done explicitly through a few lower-point examples. The general operation should follow. Note that the approach just described assumes the existence of a BCJ form in the first place. At one-loop level, one can solve all numerators using the ansatz approach of Bern, Davies, Dennen, Huang and Nohle [29] for example, which allows us to express the (pure) full Yang-Mills amplitude in BCJ form.

Granted that a dual DDM form can be obtained using the approach outlined above, the next step is to construct the corresponding dual trace form. At the moment the idea of deriving a dual trace formulation seems to be inspired to a great extend purely by academic interest. It would be natural that a dual trace form should exist if the duality is to have a group-theoretic origin. A possible practical reason is that for example when all the informations regarding to coefficients and polarizations are given, an algebraic construction of the dual trace such as the one provided in [41] should allow us to write down directly the explicit formula of the full amplitude.

To go from dual DDM form to dual trace-form, we need to find a way to rewrite basis kinematic numerators $n_{\alpha}$ into a linear combination of analogues of "single trace" and "double trace" $n_{\text {trace }}$ as what was done at tree-level [38, 40, 41]. However note that, as in the tree-level case, the number of $n_{\text {trace }}$ is much larger than $n_{\alpha}$, thus extra relations need to be manually imposed in order to solve $n_{\text {trace }}$ in terms of $n_{\alpha}$. Choosing the appropriate relations is nevertheless far from trivial, in particular one needs to avoid over-constraint and to maintain relabeling symmetry if possible. A 4-point example at one-loop level was provided by Bern and Dennen in [38], where cyclic and KK-relations were implemented. It is therefore natural to suspect that these conditions generically preserves relabeling symmetry. In this paper we generalize the result of Bern and Dennen and give a general algorithm for the construction of dual trace-form at one-loop. We will see that the solutions obtained from this algorithm indeed satisfy relabeling symmetry.

\footnotetext{
${ }^{3}$ Since we only discuss on one-loop case, we will use $n$ and $\tau$ and $\widetilde{I}$ instead of $n^{1-\text { loop }}, \tau^{1-\text { loop }}, \widetilde{I}^{1-\text { loop }}$ for convenience.

${ }^{4}$ Relevant work is in progress by the current authors.
} 


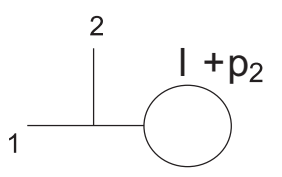

A

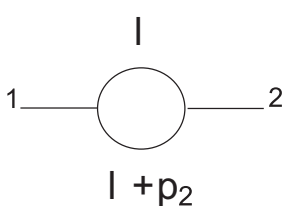

B

Figure 1. Feynman-like diagrams for two-point one-loop integrand using only cubic vertex.

This paper is organized as follows. Trough a few lower-point examples, in section 2 we demonstrate how to derive dual DDM form assuming the existence of a BCJ form. We elaborate the algorithm and calculate dual traces up to 6-points in section 3 . In section 4, we use relabeling symmetry to give another construction of dual trace-form. Finally in section 5 a brief conclusion is given.

\section{Dual DDM-form}

In this section we use explicit examples to demonstrate generically how to derive dual DDM form from BCJ form, in particular that the subtleties introduced by loop momentum degrees of freedom can be seen from the three and four point examples. As remarked in the previous section here we are assuming that all numerators has been determined using for example the method described in [29]. In the discussion below we will follow the convention where the loop momentum $l$ is defined to be the momentum carried by the propagator next to leg 1.

\subsection{Two-point example}

At two points, two diagrams $\mathrm{A}$ and $\mathrm{B}$ can be constructed using only cubic vertices, as depicted in figure 1 . The corresponding integrands are

$$
I_{A}(l)=\frac{C_{A} n_{A}(l)}{s_{12}\left(l+p_{1}+p_{2}\right)^{2}}, \quad I_{B}(l)=\frac{C_{B} n_{B}(l)}{l^{2}\left(l+p_{2}\right)^{2}} .
$$

where $C_{A}$ and $C_{B}$ are color factors

$$
C_{A}=f^{12 e} f^{e e^{\prime} e^{\prime}}, \quad C_{B}=f^{e 1 e^{\prime}} f^{e^{\prime} 2 e}
$$

and summation convention has been used implicitly. Since the structure constant is antisymmetric, $f^{e e^{\prime} e^{\prime}}=0$, we see that $C_{A}=0$. The two-point one-loop integrand becomes

$$
I_{2-p t}(l)=I_{B}(l)=n_{B}(l)\left[\frac{f^{e 1 e^{\prime}} f^{e^{\prime} 2 e}}{l^{2}\left(l+p_{2}\right)^{2}}\right] .
$$

Comparing with (1.16), we see that the part inside the bracket in above equation is simply the integrand $\widetilde{I}(1,2)$ we are looking for. It is obvious from the expression that $\widetilde{I}(1,2)$ is the one-loop integrand of color-ordered scalar theory with two external lines. 


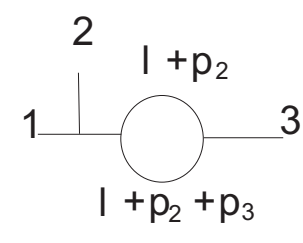

A

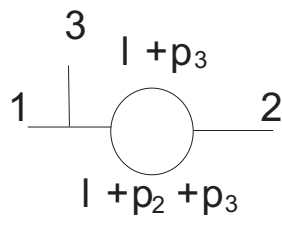

$\mathrm{B}$

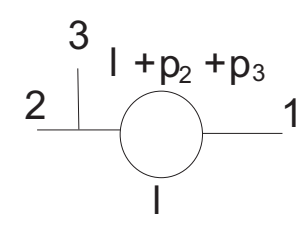

C

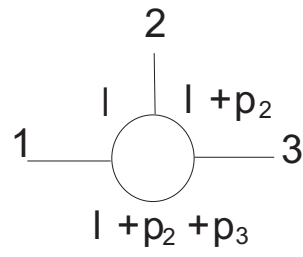

D

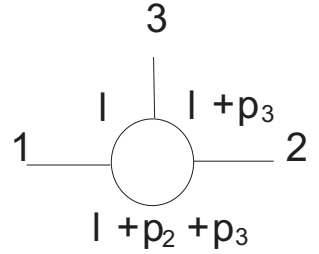

$\mathrm{E}$

Figure 2. Feynman-like diagrams for three-point one-loop integrand.

\section{$2.2 \quad$ Three-point example}

The first non-trivial case occurs at three points, where we encounter three diagram topologies (see figure 2). The first kind of topologies are the tadpole diagrams. Because of antisymmetry these diagrams do not contribute, just like the diagram A at two-points. The second kind of topologies are bubbles, i.e., diagrams A, B, C in figure 2, corresponding respectively to the three following terms

$$
\begin{aligned}
I_{A}(l) & =\frac{C_{A} n_{A}(l)}{s_{12}\left(l+p_{2}\right)^{2}\left(l+p_{2}+p_{3}\right)^{2}}, \quad I_{B}(l)=\frac{C_{A} n_{B}(l)}{s_{13}\left(l+p_{3}\right)^{2}\left(l+p_{3}+p_{2}\right)^{2}}, \\
I_{C}(l) & =\frac{C_{C} n_{C}(l)}{s_{23} l^{2}\left(l+p_{2}+p_{3}\right)^{2}} .
\end{aligned}
$$

The third kind of topologies have three lines connecting directly to the loop, i.e., diagrams $\mathrm{D}, \mathrm{E}$ in figure 2 and these contribute as

$$
I_{D}(l)=\frac{C_{D} n_{D}(l)}{l^{2}\left(l+p_{2}\right)^{2}\left(l+p_{2}+p_{3}\right)^{2}}, \quad I_{E}(l)=\frac{C_{E} n_{E}(l)}{l^{2}\left(l+p_{3}\right)^{2}\left(l+p_{3}+p_{2}\right)^{2}} .
$$

where $C_{D}, C_{E}$ are the corresponding color factors. Using Jacobi-like identity and taking $n_{D}$ and $n_{E}$ as basis, we find the following expansions

$$
n_{A}(l)=n_{D}(l)-n_{E}\left(l+p_{2}\right), \quad n_{B}(l)=n_{E}(l)-n_{D}\left(l+p_{3}\right), \quad n_{C}(l)=n_{D}(l)-n_{E}(l) .
$$

Thus $I_{A}, I_{B}, I_{C}$ can be written as

$$
\begin{aligned}
& I_{A}(l)=\frac{C_{A}\left[n_{D}(l)-n_{E}\left(l+p_{2}\right)\right]}{s_{12}\left(l+p_{2}\right)^{2}\left(l+p_{2}+p_{3}\right)^{2}}=\frac{C_{A} n_{D}(l)}{s_{12}\left(l+p_{2}\right)^{2}\left(l+p_{2}+p_{3}\right)^{2}}-\frac{C_{A} n_{E}(l)}{s_{12} l^{2}\left(l+p_{3}\right)^{2}}+T_{A} \\
& I_{B}(l)=\frac{C_{B}\left[n_{E}(l)-n_{D}\left(l+p_{3}\right)\right]}{s_{13}\left(l+p_{3}\right)^{2}\left(l+p_{3}+p_{2}\right)^{2}}=\frac{C_{B} n_{E}(l)}{s_{13}\left(l+p_{3}\right)^{2}\left(l+p_{3}+p_{2}\right)^{2}}-\frac{C_{B} n_{D}(l)}{s_{13}(l)^{2}\left(l+p_{2}\right)^{2}}+T_{B} \\
& I_{C}(l)=\frac{C_{C}\left[n_{D}(l)-n_{E}(l)\right]}{s_{23} l^{2}\left(l+p_{2}+p_{3}\right)^{2}}
\end{aligned}
$$


Note that in order to be able to extract $n_{i}(l)$ instead of shifted numerators we simultaneously add and subtract unshifted terms. The differences between shifted and unshifted terms, $T_{A}$ and $T_{B}$, integrated to give zero.

$$
\begin{aligned}
& T_{A}=\frac{C_{A}}{s_{12}}\left[\frac{n_{E}(l)}{l^{2}\left(l+p_{3}\right)^{2}}-\frac{n_{E}\left(l+p_{2}\right)}{\left(l+p_{2}\right)^{2}\left(l+p_{2}+p_{3}\right)^{2}}\right], \\
& T_{B}=\frac{C_{B}}{s_{13}}\left[\frac{n_{D}(l)}{l^{2}\left(l+p_{2}\right)^{2}}-\frac{n_{D}\left(l+p_{3}\right)}{\left(l+p_{3}\right)^{2}\left(l+p_{3}+p_{2}\right)^{2}}\right] .
\end{aligned}
$$

Therefore up to terms vanish upon integration, the full integrand is given as

$$
\begin{aligned}
I(1,2,3)(l)= & n_{D}(l)\left[\frac{C_{A}}{s_{12}\left(l+p_{2}\right)^{2}\left(l+p_{2}+p_{3}\right)^{2}}-\frac{C_{B}}{s_{13}(l)^{2}\left(l+p_{2}\right)^{2}}+\frac{C_{C}}{s_{23} l^{2}\left(l+p_{2}+p_{3}\right)^{2}}\right. \\
& \left.+\frac{C_{D}}{l^{2}\left(l+p_{2}\right)^{2}\left(l+p_{2}+p_{3}\right)^{2}}\right] \\
& +n_{E}(l)\left[-\frac{C_{A}}{s_{12} l^{2}\left(l+p_{3}\right)^{2}}+\frac{C_{B}}{s_{13}\left(l+p_{3}\right)^{2}\left(l+p_{3}+p_{2}\right)^{2}}-\frac{C_{C}}{s_{23} l^{2}\left(l+p_{2}+p_{3}\right)^{2}}\right. \\
& \left.+\frac{C_{E}}{l^{2}\left(l+p_{3}\right)^{2}\left(l+p_{3}+p_{2}\right)^{2}}\right] \equiv n_{D}(l) \widetilde{I}(1,2,3)(l)+n_{E}(l) \widetilde{I}(1,3,2)(l), \quad(2.9)
\end{aligned}
$$

The above is exactly the expected dual DDM form of one-loop amplitude (1.16), where $n_{D}$ and $n_{E}$ are just the kinematic basis numerators. The corresponding expressions for $\widetilde{I}(1,2,3)(l)$ and $\widetilde{I}(1,3,2)(l)$ are again the three-point one-loop integrands of color-ordered scalar theory.

\section{$2.3 \quad$ Four-point example}

At four-points, four types of diagrams are present. Except for tadpoles, which vanish because antisymmetry, the rest of the diagrams are list in figures 3,4 and 5. We choose D.1-D. 6 of figure 5 to be the basis numerators $n_{\alpha}$ and expand those in figure 3 and figure 4 using Jacobi identities. The coefficient of $n_{1234}^{\mathrm{DDM}}$ for example receives contributions from diagrams A.1, A.3, B.1, B.3, B.5, B.8, B.9, B.10, B.12, C.1, C.5, C.8, C.12, D.1, and is given as

$$
\begin{aligned}
& \frac{C_{A .1}}{s_{12} s_{34}\left(l+p_{2}\right)^{2}\left(l-p_{1}\right)^{2}}+\frac{C_{A .3}}{s_{14} s_{23}\left(l+p_{4}\right)^{2}\left(l-p_{1}\right)^{2}} \\
& +\frac{C_{B .1}}{s_{12} s_{123}\left(l+p_{2}+p_{3}\right)^{2}\left(l-p_{1}\right)^{2}}+\frac{C_{B .3}}{s_{23} s_{123}\left(l+p_{2}+p_{3}\right)^{2}\left(l-p_{1}\right)^{2}}+\frac{C_{B .5}}{s_{14} s_{124}\left(l+p_{2}+p_{4}\right)^{2}\left(l-p_{1}\right)^{2}} \\
& +\frac{C_{B .7}}{s_{13} s_{134}\left(l+p_{3}+p_{4}\right)^{2}\left(l-p_{1}\right)^{2}}+\frac{C_{B .10}}{s_{34} s_{134}\left(l+p_{3}+p_{4}\right)^{2}\left(l-p_{1}\right)^{2}}+\frac{C_{23} s_{234} l^{2}\left(l-p_{1}\right)^{2}}{s_{B .12}} \\
& +\frac{C_{C .1}}{s_{34} s_{234} l^{2}\left(l-p_{1}\right)^{2}}+\frac{C_{12}\left(l+p_{2}\right)^{2}\left(l+p_{2}+p_{3}\right)^{2}\left(l-p_{1}\right)^{2}}{s_{14}}+\frac{C_{C .12}\left(l+p_{4}\right)^{2}\left(l+p_{2}+p_{4}\right)^{2}\left(l-p_{1}\right)^{2}}{s_{14}} \\
& +\frac{C_{C .1}}{s_{23} l^{2}\left(l+p_{2}+p_{3}\right)^{2}\left(l-p_{1}\right)^{2}}+\frac{C_{D_{1}}}{s_{34} l^{2}\left(l+p_{2}\right)^{2}\left(l-p_{1}\right)^{2}}+\frac{p_{1}\left(l+p_{3}\right)^{2}\left(l+p_{3}+p_{4}\right)^{2}\left(l-p_{1}\right)^{2}}{l^{2}(l)}
\end{aligned}
$$




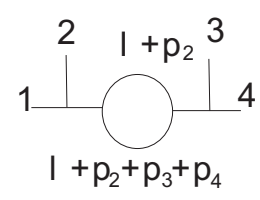

A. 1

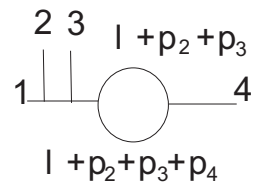

B.1

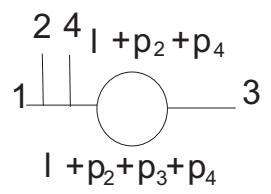

B. 4

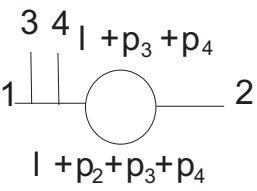

B. 7

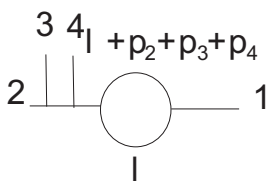

B. 10

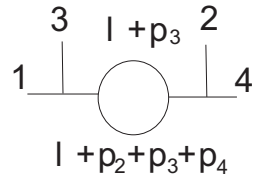

A. 2

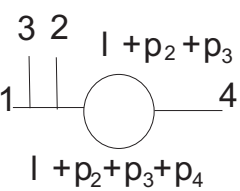

B. 2

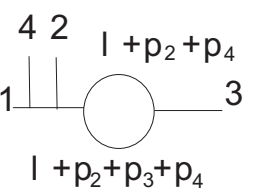

B. 5

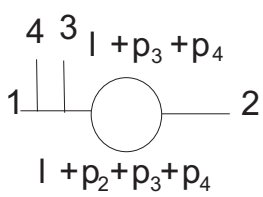

B. 8

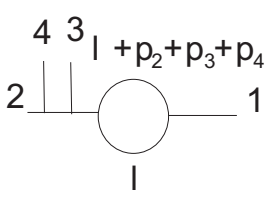

B. 11

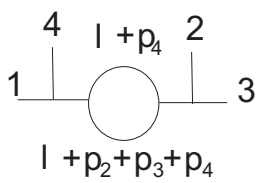

A. 3

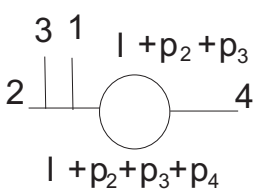

B. 3

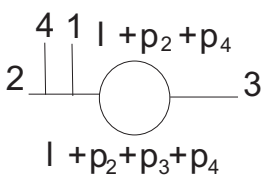

B. 6

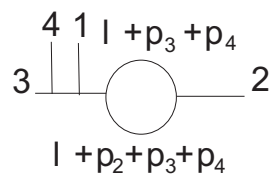

B.9

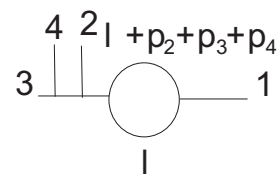

B. 12

Figure 3. Feynman-like diagrams with two lines connected to the loop in four-point case.

where we have neglected terms that integrate to zero. We recognize the above expression as the four-point one-loop integrand $\widetilde{I}(1,2,3,4)$ of color-ordered scalar theory. Repeating similar operations on the rest of the terms and we do get the expected dual DDM form (1.16)

$$
I(1,2,3,4)(l)=\sum_{\sigma \in \text { permutations of }\{2,3,4\}} n_{1, \sigma}(l) \widetilde{I}(1, \sigma) .
$$

up to terms that vanish after loop integration, where each $\widetilde{I}(1, \sigma)$ can be identified as the colored scalar integrand at one-loop level. The above procedure generalizes to higher points although the computations will become much more complicated. 


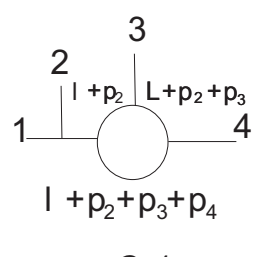

C. 1
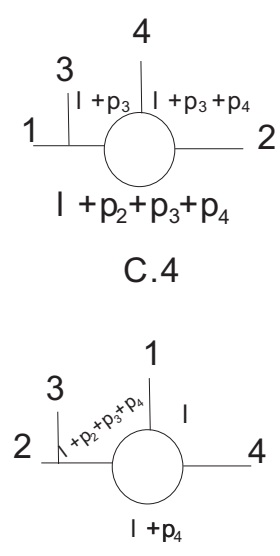

C. 7

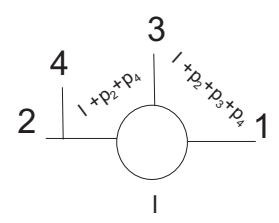

C. 10

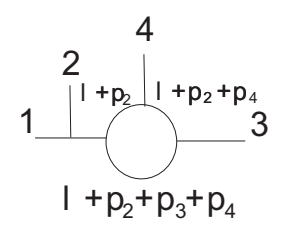

C. 2

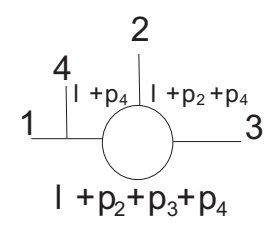

C. 5

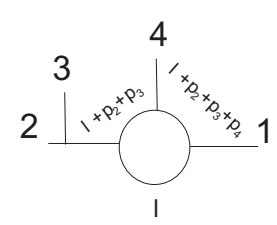

C. 8

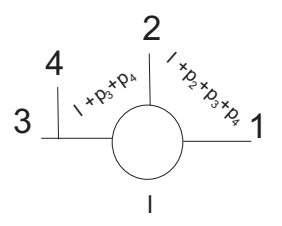

C. 11

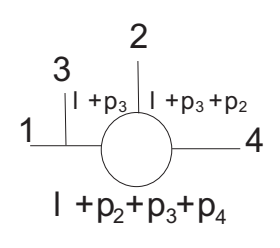

C. 3

Figure 4. Feynman-like diagrams with three lines connected to the loop in four-point case.

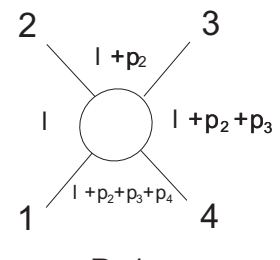

D. 1

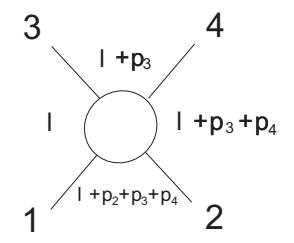

D. 4

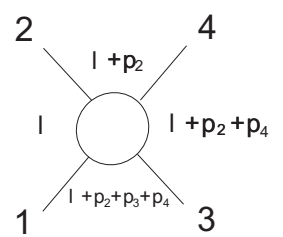

D.2

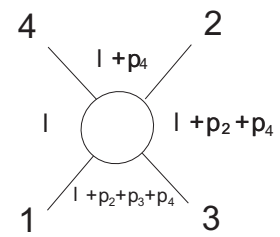

D.5

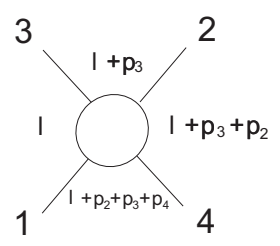

D.3

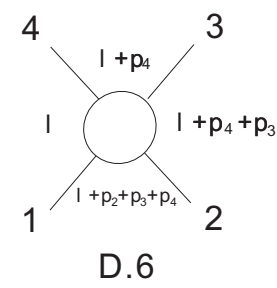

Figure 5. Feynman-like diagrams with four lines connected to the loop in four-point case. 


\section{Dual trace-form}

In the discussions above we saw that the dual DDM-form can be derived through relatively straightforward manipulations. Deriving a corresponding dual trace-form at one-loop however turns out to be less direct, especially because of the extra conditions required to define dual trace factors $[38,40]$.

Recall that at tree-level, the set of numerators $n_{1 \sigma n}$, consisting of $(n-2)$ ! elements, having legs 1 and $n$ fixed at two ends, were translated into $(n-1)$ ! dual traces $\tau_{1 \widetilde{\sigma}}$, which are counterparts of the single color trace factors. To uniquely determine $\tau$ we need to impose KK-relation among $\tau_{1 \widetilde{\sigma}}$ 's, so the number of independent dual traces can be reduced to $(n-2)$ !. The algorithm formally picks a fixed pair $(1, n)$ to define basis numerators. To examine if the solution satisfy relabling symmetry we need to inspect the transformation under permutaions of legs 1 and $n$.

At one-loop level, similar constraints are required to properly define dual traces. For the purpose of discussion let us first review the $\mathrm{U}\left(N_{c}\right)$ color structure at one-loop, which also serves as input to the definition of dual traces.

\subsection{General structure of the defining conditions}

Generically, the color factors appear in the DDM-form at one-loop level can be translated into double trace factors,

$$
\begin{aligned}
c_{\{\sigma\}}^{1 \text {-loop }} & =f^{x_{1} \sigma_{1} x_{2}} f^{x_{2} \sigma_{2} x_{3}} \ldots f^{x_{n} \sigma_{n} x_{1}}=\operatorname{Tr}\left(T^{x_{1}}\left[T^{\sigma_{1}},\left[T^{\sigma_{2}}, \ldots\left[T^{\sigma_{n}}, T^{x_{1}}\right]\right]\right]\right) \\
& =\sum_{\sigma \in O P(\{\alpha\} \bigcup\{\beta\})}(-1)^{n_{\beta}} \operatorname{Tr}\left(T^{x_{1}} T^{\alpha_{1}} \ldots T^{\alpha_{n_{\alpha}}} T^{x_{1}} T^{\beta_{n_{\beta}}} \ldots T^{\beta_{1}}\right) \\
& =\sum_{\sigma \in O P(\{\alpha\} \bigcup\{\beta\})}(-1)^{n_{\beta}} \operatorname{Tr}\left(T^{\alpha_{1}} \ldots T^{\alpha_{n_{\alpha}}}\right) \operatorname{Tr}\left(T^{\beta_{n_{\beta}}} \ldots T^{\beta_{1}}\right),
\end{aligned}
$$

where in the last line we used the property of $\mathrm{U}\left(N_{c}\right)$,

$$
\sum_{x_{1}} \operatorname{Tr}\left(T^{x_{1}} A T^{x_{1}} B\right)=\operatorname{Tr}(A) \operatorname{Tr}(B) .
$$

Note however, two exceptional cases call for special attention. When the repeated generators are adjacent, single trace factors are produced instead. This can happen in equation (3.1) as

$$
\sum_{x_{1}} \operatorname{Tr}\left(T^{x_{1}} T^{\sigma_{1}} \ldots T^{\sigma_{n}} T^{x_{1}}\right)=N_{c} \operatorname{Tr}\left(T^{\sigma_{1}} \ldots T^{\sigma_{n}}\right)
$$

or as

$$
(-)^{n} \sum_{x_{1}} \operatorname{Tr}\left(T^{x_{1}} T^{x_{1}} T^{\sigma_{n}} T^{\sigma_{n-1}} \ldots T^{\sigma_{1}}\right)=(-)^{n} N_{c} \operatorname{Tr}\left(T^{\sigma_{n}} T^{\sigma_{n-1}} \ldots T^{\sigma_{1}}\right)
$$

Inspired by the above algebraic structure, it is natural to assume that there are kinematic correspondence of the following color trace factors

$$
\operatorname{Tr}\left(T^{\alpha_{1}} \ldots T^{\alpha_{m}}\right) \operatorname{Tr}\left(T^{\beta_{1}} \ldots T^{\beta_{n}}\right) \rightarrow \tau_{\alpha ; \beta}, \quad \operatorname{Tr}\left(T^{\sigma_{1}} \ldots T^{\sigma_{n}}\right) \rightarrow \tau_{\sigma},
$$


where kinematic trace factors $\tau_{\alpha ; \beta}$ and $\tau_{\alpha}$ are cyclic invariant. Thus we can impose the following commutation-like relation between $n_{\alpha}$ in the dual DDM-form and kinematic trace structure $\tau_{\alpha ; \beta}$ in the dual trace-form as

$$
n_{\{\sigma\}}^{1-\text { loop }}=\sum_{\sigma \in O P(\{\alpha\} \bigcup\{\beta\})}(-1)^{n_{\beta}} \tau_{\alpha ; \beta^{T}},
$$

where $\beta^{T}$ should be understood as the ordered set $\beta$ with its ordering reversed. Once a dual DDM form is written down, relation (3.6) alone allows us to translate it into the dual trace form introduced at the very beginning of this paper,

$$
\begin{aligned}
\mathcal{A}_{1-\text { loop }}= & i g^{n} \int \frac{d^{D} l}{(2 \pi)^{D}} \sum_{\sigma \in S_{n-1}} n_{1, \sigma}^{\mathrm{DDM}}(l) \widetilde{I}(1, \sigma) \\
= & i g^{n} \int \frac{d^{D} l}{(2 \pi)^{D}} \sum_{m=0}^{\lfloor n / 2\rfloor} \sum_{\sigma \in S_{n} / S_{n ; m}} \tau_{\left\{\sigma_{1}, \ldots, \sigma_{n-m}\right\},\left\{\sigma_{n-m+1}, \ldots, \sigma_{n}\right\}}(l) \\
& \times \widetilde{I}\left(\left\{\sigma_{1}, \ldots, \sigma_{n-m}\right\} ;\left\{\sigma_{n-m+1}, \ldots, \sigma_{n}\right\}\right) .
\end{aligned}
$$

Generically the dual traces can depend on loop momentum, however since that all loop momenta appear in the dual DDM form are already properly aligned (in the sense that no shifted numerator is present), we see that going from the first line to the second line of equation (3.7) follows the same operation on color factors in a rather straightforward manner. As it happens to the color factors, when applying (3.6) we have two special cases corresponding to those when the repeated indices are adjacent, and we retain strictly the same correspondence between traces of $\mathrm{U}\left(N_{c}\right)$ and $\tau$ by writing: when $\alpha=\emptyset, \tau_{\alpha ; \beta^{T}} \rightarrow$ $N_{c} \tau_{\beta^{T}}$, and when $\beta=\emptyset, \tau_{\alpha ; \beta^{T}} \rightarrow N_{c} \tau_{\alpha}$. In the absence of an algebraic interpretation, we are treating the "kinematic rank $N_{c}$ " here as a remaining degree of freedom left by equation (3.6).

From equation (3.6), one can see that the number of all $\tau$ together clearly exceeds that of $n$. In total there are $(n-1) ! / 2$ independent $n_{\alpha}$ 's because $n_{\alpha}$ is cyclic invariant and satisfies the following reflection relation

$$
n_{\alpha}=(-)^{M} n_{\alpha^{T}}
$$

where $M$ is the number of elements of the set $\alpha$. To be able to solve $\tau$ in terms of $n_{\alpha}$, we need to impose extra equations. Nevertheless without any clue from an actual kinematic algebra it is rather difficult to pick the proper constraint that does not spoil relabeling symmetry. A possible solution was proposed by Bern and Dennen in [38], where KleissKuijf (KK) relation was imposed on $N=4$ super Yang-Mills amplitude at 4-points. In the light of this initial success we are assuming the same KK relation between the kinematic single and double traces

$$
\tau_{\alpha ; \beta}=(-)^{n_{\beta}} \sum_{C \in Z_{n_{\beta}}} \sum_{\sigma \in \mathcal{C O P}\left(\alpha \cup C(\beta)^{T}\right)} \tau_{\sigma}
$$

generalizes to higher points, in the hope that relabeling symmetry can be preserved despite that $\mathrm{U}\left(N_{c}\right)$ traces generically do not satisfy the same relation. Here we use $\mathcal{C O} \mathcal{P}$ to denote 
"cyclic ordered permutations", in the sense that we include all possible permutations where the cyclic orderings of both $\alpha$ and $C(\beta)^{T}$ are kept fixed. It is worth noticing that subsets $\alpha$ and $\beta$ in equation (3.9) were not treated on equal footing. For this reason we manually impose the following two additional conditions.

$$
\tau_{\alpha ; \beta}=\tau_{\beta ; \alpha},
$$

and

$$
\tau_{\alpha}=(-1)^{M} \tau_{\alpha^{T}},
$$

where $M$ is the number of elements of the set $\alpha$. The minus sign in the reflection condition (3.11) is necessary in order to be consistent with (3.9) and (3.10).

Equations (3.9), (3.10) and (3.11) together reduces the number of independent dual trace factors to $(n-1) ! / 2$. Under these constraints the original commutation-like equations (3.6) become an $(n-1) ! / 2$ by $(n-1) ! / 2$ matrix equation,

$$
n_{1 \sigma}=\sum_{\sigma^{\prime} \in S_{n-1}} G\left[\sigma \mid \sigma^{\prime}\right] \tau_{1 \sigma^{\prime}}
$$

Once the matrix $G$ is known, in principle we can solve $\tau_{1 \sigma^{\prime}}$ in terms of the numerators $n_{1 \sigma}$ and in turn determine all the kinematic trace factors.

General algorithm. To summarize, the general algorithm of constructing kinematic trace factors is given by the following:

- Starting with any dual-DDM basis numerator $n_{1, \ldots, n}$ we consider all possible splittings of its label $\{1, \ldots, n\}$ into two subsets $\alpha$ and $\beta$, each can be empty. Generically there will be $2^{n}$ splittings. For example at four-points, denoting the one-loop dual-DDM factor as $n_{1, \sigma}^{1-\text { loop }}, \sigma \in \operatorname{perm}(2,3,4)$. the relation between $n$ and $\tau$ is given by

$$
\begin{aligned}
n_{1234}^{1-\text { loop }}= & N_{c} \tau_{\{1234\}}-\tau_{\{234\},\{1\}}-\tau_{\{134\},\{2\}}-\tau_{\{124\},\{3\}}-\tau_{\{123\},\{4\}} \\
& +\tau_{\{34\},\{21\}}+\tau_{\{24\},\{31\}}+\tau_{\{23\},\{41\}}+\tau_{\{13\},\{42\}}+\tau_{\{14\},\{32\}}+\tau_{\{12\},\{43\}} \\
& -\tau_{\{4\},\{321\}}-\tau_{\{3\},\{421\}}-\tau_{\{2\},\{431\}}-\tau_{\{1\},\{432\}}+N_{c} \tau_{\{4321\}}
\end{aligned}
$$

- We then impose KK relation (3.9) on $\tau$. In the four-point case, we have

$$
\begin{aligned}
& \tau_{\{b c d\},\{a\}}=-\tau_{\{a b c d\}}-\tau_{\{b a c d\}}-\tau_{\{b c a d\}}, \\
& \tau_{\{c d\},\{b a\}}=\tau_{\{a b c d\}}+\tau_{\{a c b d\}}+\tau_{\{c a b d\}}+\tau_{\{b a c d\}}+\tau_{\{b c a d\}}+\tau_{\{c b a d\}}, \\
& \tau_{\{d\},\{c b a\}}=-\tau_{\{a b c d\}}-\tau_{\{a b d c\}}-\tau_{\{a d b c\}},
\end{aligned}
$$

Substituting these relations into (3.13) and using cyclic symmetry $\tau_{\{a b c d\}}=\tau_{\{d a b c\}}$, we get

$$
\begin{aligned}
n_{1234}^{1-\text { loop }}= & \left(15+N_{c}\right) \tau_{\{1234\}}+10 \tau_{\{1243\}}+10 \tau_{\{1324\}}+10 \tau_{\{1342\}}+10 \tau_{\{1423\}} \\
& +\left(5+N_{c}\right) \tau_{\{1432\}} .
\end{aligned}
$$


- Using reflection relation (3.11) we can reduce the obtained equations further. For example, above equation is reduced to

$$
n_{1234}^{1-\text { loop }}=\left(20+2 N_{c}\right) \tau_{1234}+20 \tau_{1243}+20 \tau_{1324},
$$

Repeating the same manipulations for all basis numerators, we arrive at the matrix equation (3.12), from which we can solve for all dual traces.

The $G$ matrix. Now we discuss the computation of the $G$ matrix. The calculation can be divided into two steps. The first step is to calculate extended $\widetilde{G}$-matrix $\widetilde{G}[\sigma \mid \rho]$ where $\sigma, \rho \in S_{n} / Z_{n}$ (i.e., all permutations up to cyclic ordering). The second step is to impose the reflection relation (3.11), i.e,

$$
G[\sigma \mid \rho]=\widetilde{G}[\sigma \mid \rho]+(-)^{n} \widetilde{G}\left[\sigma \mid \rho^{T}\right], \quad \sigma, \rho \in\left(S_{n} / Z_{n}\right) / Z_{2}
$$

Since the second step is easy, we will focus on the first step, i.e., the extended $\widetilde{G}$-matrix. Elements of extended $\widetilde{G}$-matrix depend on $N_{c}$ only for following two kinds of structures

$$
\widetilde{G}[\sigma \mid \sigma]=a_{0}+N_{c}, \quad \widetilde{G}\left[\sigma \mid \sigma^{T}\right]=(-)^{n}\left(b_{0}+N_{c}\right)
$$

where $a_{0}, b_{0}$ are constants. Because this dependence, if we know the extended $\widetilde{G}$-matrix for $N_{c}=1$, we will know the extended $\widetilde{G}$-matrix for general $N_{c}$.

To demonstrate the calculation of element $\widetilde{G}[\sigma \mid \rho]$, let us use four-point result (3.15) with $N_{c}=1$ as an example. For this example, we have $\sigma=\{1,2,3,4\}$ fixed and $3 !=6$ different choices of $\rho$. Given the ordering of $\sigma$, there are $2^{4}=16$ different splittings to two subsets. Among them, 8 of them with 1 at the first subset are given by (remembering to keep relative ordering)

$$
\begin{aligned}
& \{1,2,3,4\} \rightarrow\left(\sigma_{L}, \sigma_{R}\right) \\
& =(1234, \emptyset) /(123,4) /(124,3) /(134,2) /(12,34) /(13,24) /(14,23) /(1,234)
\end{aligned}
$$

and other 8 are obtained by exchanging these two subsets. We do similar splitting to the ordering $\rho$, but now we will allow the cyclic shifting of one subset. For example, with $\rho=\{1,2,4,3\}$ we will have following splitting with 1 at the first position of the first subset (by cyclic symmetry, we can always fix one element)

$$
\begin{aligned}
\{1,2,4,3\} \rightarrow\left(\rho_{L}, \rho_{R}\right)= & (1243, \emptyset) /(124,3) /(123,4) /(143,2) /(12,43) /(12,34) /(14,23) \\
& /(14,32) /(13,24) /(13,42) /(1,243) /(1,432) /(1,324)
\end{aligned}
$$

where since we have fixed 1 , we have to include the cyclic shifting of $\rho_{R}$. Comparing these two splitting (3.19) and (3.20), we see that there are five splittings to be same:

$$
(123,4) /(124,3) /(12,34) /(13,24) /(14,23) \Longrightarrow \widetilde{G}[\{1,2,3,4\} \mid\{1,2,4,3\}]=2 \times 5=10
$$

where factor 2 comes from exchanging of two subsets. One can easily check that all other five coefficients in (3.15) can be obtained by same way. For $\rho=\{1,2,3,4\}$ there are $8 \times 2$ splitting. For $\rho=\{1,3,2,4\},(124,3) /(134,2) /(12,34) /(13,24) /(14,23)$ from $(3.19)$ 
are taken, so $5 \times 2=10$. For $\rho=\{1,3,4,2\},(134,2) /(12,34) /(13,24) /(14,23) /(1,234)$ from (3.19) are taken, so $5 \times 2=10$. For $\rho=\{1,4,2,3\},(123,4) /(12,34) /(13,24)$ $/(14,23) /(1,234)$ from $(3.19)$ are taken, so $5 \times 2=10$. Finally for $\rho=\{1,4,3,2\}$, $(12,34) /(13,24) /(14,23)$ from $(3.19)$ are taken, so $3 \times 2=6$.

Having about general discussions, now we demonstrate our algorithm by several examples.

\section{2 $\quad$ Four-point dual traces}

Under our imposed conditions (3.9), (3.10) and (3.11) the number of independent $n$ 's and $\tau^{\prime}$ 's is $\frac{(4-1) !}{2}=3$. We take the liberty to choose following three orderings (1234), (1243) and (1324) as our basis. Using our algorithm for $G$-matrix, equation (3.12) yields

$$
\begin{aligned}
& n_{1234}^{1 \text {-loop }}=\left(20+2 N_{c}\right) \tau_{1234}+20 \tau_{1243}+20 \tau_{1324}, \\
& n_{1243}^{1-\text { loop }}=20 \tau_{1234}+\left(20+2 N_{c}\right) \tau_{1243}+20 \tau_{1324}, \\
& n_{1324}^{1-\text { loop }}=20 \tau_{1234}+22 \tau_{1243}+\left(20+2 N_{c}\right) \tau_{1324} .
\end{aligned}
$$

The determinant of $G$-matrix is $\operatorname{det}(G)=8 N_{c}^{2}\left(30+N_{c}\right)$ for generic $N_{c}$, from which we derive the solution for $\tau_{1234}$,

$$
\tau_{1234}=\frac{1}{2 N_{c}\left(30+N_{c}\right)}\left(\left(20+N_{c}\right) n_{1234}-10 n_{1243}-10 n_{1324}\right) .
$$

Expressions of other orderings $\tau_{\rho}$ can be obtained by relabeling symmetry.

This result seems to differ from the result previously obtained for $N=4 \mathrm{SYM}$ theory in [38]. To connect the two results, notice that for $N=4$ SYM, only have box diagrams contribute and the corresponding $n$ is given as

$$
n_{a b c d}^{1-\text { loop }}=s_{a b} s_{a d} A^{\text {tree }}(a, b, c, d) .
$$

Substituting (3.24) into (3.23) and using tree-level amplitude relation to write all the fourpoint tree amplitudes in terms of $A(1234)$, we get

$$
\tau_{1234}^{1-\text { loop }}=\frac{1}{62} s t A^{\text {tree }}(1,2,3,4) .
$$

which is just the result given by Bern and Dennen when $N_{c}$ is chosen to be 1 .

Our result (3.23) has a free parameter $N_{c}$. It is easy to see that $N_{c}$ can not be 0 or -30 because for these two values, determinant of $G$-matrix is zero, i.e., $G$-matrix is degenerated and we can not solve $\tau$ by $n_{\alpha}$. Also, with particular choice of $N_{c}$, we may get simpler expressions. For example, when $N_{c}=-10$ we get

$$
\tau_{1234}=\frac{-1}{40}\left(+n_{1234}-n_{1243}-n_{1324}\right)
$$

while when $N_{c}=-20$ we get

$$
\tau_{1234}=\frac{1}{40}\left(n_{1243}+n_{1324}\right)
$$




\subsection{Five-point case}

Let us apply the same algorithm to five points. The relation between DDM basis numerator $n$ and dual trace $\tau$ is given as

$$
\begin{aligned}
n_{a_{1} a_{2} a_{3} a_{4} a_{5}=} & \left(30+2 N_{c}\right) \tau_{\left\{a_{1} a_{2} a_{3} a_{4} a_{5}\right\}}+12 \tau_{\left\{a_{1} a_{2} a_{3} a_{5} a_{4}\right\}}+12 \tau_{\left\{a_{1} a_{2} a_{4} a_{3} a_{5}\right\}}+12 \tau_{\left\{a_{1} a_{3} a_{2} a_{4} a_{5}\right\}} \\
& +6 \tau_{\left\{a_{1} a_{2} a_{4} a_{5} a_{3}\right\}}+6 \tau_{\left\{a_{1} a_{2} a_{5} a_{3} a_{4}\right\}}+6 \tau_{\left\{a_{1} a_{3} a_{4} a_{2} a_{5}\right\}}+6 \tau_{\left\{a_{1} a_{4} a_{2} a_{3} a_{5}\right\}} \\
& -12 \tau_{\left\{a_{1} a_{2} a_{5} a_{4} a_{3}\right\}}-12 \tau_{\left\{a_{1} a_{4} a_{3} a_{2} a_{5}\right\}}-6 \tau_{\left\{a_{1} a_{3} a_{2} a_{5} a_{4}\right\}}+0 \tau_{\left\{a_{1} a_{3} a_{5} a_{2} a_{4}\right\}}
\end{aligned}
$$

The number of independent $n \mathrm{~s}$ and $\tau \mathrm{s}$ is $(5-1) ! / 2=12$. We choose the our basis to be (12345), (12354), (12435), (12453), (12434), (12543), (13245), (13254), (13425), (13524), (14235), (14325) in order, which leads to following matrix $G$

$$
G=\left(\begin{array}{cccccccccccc}
2 \mathrm{Nc}+30 & 12 & 12 & 6 & 6 & -12 & 12 & -6 & 6 & 0 & 6 & -12 \\
12 & 2 \mathrm{Nc}+30 & 6 & -12 & 12 & 6 & -6 & 12 & 0 & 6 & 12 & -6 \\
12 & 6 & 2 \mathrm{Nc}+30 & 12 & -12 & 6 & 6 & 0 & -12 & 6 & 12 & 6 \\
6 & -12 & 12 & 2 \mathrm{Nc}+30 & 6 & 12 & 12 & -6 & -6 & -12 & -6 & 0 \\
6 & 12 & -12 & 6 & 2 \mathrm{Nc}+30 & 12 & 0 & 6 & 6 & -12 & -6 & -12 \\
-12 & 6 & 6 & 12 & 12 & 2 \mathrm{Nc}+30 & -6 & 12 & -12 & -6 & 0 & 6 \\
12 & -6 & 6 & 12 & 0 & -6 & 2 \mathrm{Nc}+30 & 12 & 12 & 6 & -12 & 6 \\
-6 & 12 & 0 & -6 & 6 & 12 & 12 & 2 \mathrm{Nc}+30 & 6 & 12 & -6 & 12 \\
6 & 0 & -12 & -6 & 6 & -12 & 12 & 6 & 2 \mathrm{Nc}+30 & -12 & 6 & 12 \\
0 & 6 & 6 & -12 & -12 & -6 & 6 & 12 & -12 & 2 \mathrm{Nc}+30 & -12 & -6 \\
6 & 12 & 12 & -6 & -6 & 0 & -12 & -6 & 6 & -12 & 2 \mathrm{Nc}+30 & 12 \\
-12 & -6 & 6 & 0 & -12 & 6 & 6 & 12 & 12 & -6 & 12 & 2 \mathrm{Nc}+30
\end{array}\right)
$$

with determinant $\operatorname{det}(G)=2^{12} N_{c}^{6}\left(N_{c}+30\right)^{6}$. Therefore the solution is

$$
\begin{aligned}
\tau_{\{12345\}}= & \frac{1}{2 N_{c}\left(30+N_{c}\right)}\left\{\left(15+N_{c}\right) n_{12345}-6 n_{12354}-6 n_{12435}-3 n_{12453}\right. \\
& \left.-3 n_{12534}+6 n_{12543}-6 n_{13245}+3 n_{13254}-3 n_{13425}-3 n_{14235}+6 n_{14325}\right\}
\end{aligned}
$$

Other $\tau$ 's can be obtained using relabeling symmetry. For this expression, if we choose $N_{c}=-15$, all coefficients are $\frac{ \pm 2}{150}$ and $\frac{1}{150}$. Especially the first coefficients $\left(15+N_{c}\right) \rightarrow 0$.

\subsection{Six-point example}

At six-points, the basis can be labeled by the following $(6-1) ! / 2=60$ orderings

$\{1,2,3,4,5,6\},\{1,2,3,4,6,5\},\{1,2,3,5,4,6\},\{1,2,3,5,6,4\},\{1,2,3,6,4,5\},\{1,2,3,6,5,4\}$, $\{1,2,4,3,5,6\},\{1,2,4,3,6,5\},\{1,2,4,5,3,6\},\{1,2,4,5,6,3\},\{1,2,4,6,3,5\},\{1,2,4,6,5,3\}$, $\{1,2,5,3,4,6\},\{1,2,5,3,6,4\},\{1,2,5,4,3,6\},\{1,2,5,4,6,3\},\{1,2,5,6,3,4\},\{1,2,5,6,4,3\}$, $\{1,2,6,3,4,5\},\{1,2,6,3,5,4\},\{1,2,6,4,3,5\},\{1,2,6,4,5,3\},\{1,2,6,5,3,4\},\{1,2,6,5,4,3\}$, $\{1,3,2,4,5,6\},\{1,3,2,4,6,5\},\{1,3,2,5,4,6\},\{1,3,2,5,6,4\},\{1,3,2,6,4,5\},\{1,3,2,6,5,4\}$, $\{1,3,4,2,5,6\},\{1,3,4,2,6,5\},\{1,3,4,5,2,6\},\{1,3,4,6,2,5\},\{1,3,5,2,4,6\},\{1,3,5,2,6,4\}$, $\{1,3,5,4,2,6\},\{1,3,5,6,2,4\},\{1,3,6,2,4,5\},\{1,3,6,2,5,4\},\{1,3,6,4,2,5\},\{1,3,6,5,2,4\}$, $\{1,4,2,3,5,6\},\{1,4,2,3,6,5\},\{1,4,2,5,3,6\},\{1,4,2,6,3,5\},\{1,4,3,2,5,6\},\{1,4,3,2,6,5\}$, $\{1,4,3,5,2,6\},\{1,4,3,6,2,5\},\{1,4,5,2,3,6\},\{1,4,5,3,2,6\},\{1,4,6,2,3,5\},\{1,4,6,3,2,5\}$, $\{1,5,2,3,4,6\},\{1,5,2,4,3,6\},\{1,5,3,2,4,6\},\{1,5,3,4,2,6\},\{1,5,4,2,3,6\},\{1,5,4,3,2,6\} .(3.31)$ 
The expansion coefficients of $n_{123456}$ into $\tau$ s, i.e, $G[\{123456\} \mid \rho]=G_{1 i} i=1, \ldots, 60$ respectively by the orderings listed above, is given by

$$
\begin{aligned}
G_{1 i}=\{ & 62+2 N_{c}, 34,34,22,22,12,34,22,22,22, \\
& 18,16,22,18,12,16,26,22,22,16, \\
& 16,22,22,34,34,22,22,16,16,22, \\
& 22,16,22,18,18,12,16,18,18,18, \\
& 12,18,22,16,18,12,12,22,16,18, \\
& 26,22,18,18,22,16,16,22,22,34\},
\end{aligned}
$$

Other $G_{i j}$ can be obtained by relabeling symmetry. The determinant of matrix $G$ is

$$
\operatorname{det}(G)=2^{60} N_{c}^{24}\left(N_{c}+18\right)^{5}\left(N_{c}+21\right)^{16}\left(N_{c}+56\right)^{9}\left(N_{c}+60\right)^{5}\left(N_{c}+630\right),
$$

and the solution is given by

$$
\tau_{\sigma}=G^{-1}[\sigma \mid \rho] n_{\rho}
$$

The inverse of matrix $G$ is very complicated, but with relabeling symmetry, it is enough to give the first row, i.e., $G_{1 i}^{-1}$ with $i=1, \ldots, 60$. To have a feeling about the $N_{c^{-}}$dependence, we list all 60 elements as following:

$$
\begin{aligned}
G_{11,12,13,14,15}^{-1}\{ & \frac{1}{120}\left(\frac{5}{N_{c}+18}+\frac{16}{N_{c}+21}+\frac{9}{N_{c}+56}+\frac{5}{N_{c}+60}+\frac{1}{N_{c}+630}+\frac{24}{N_{c}}\right), \\
& \frac{1}{2520}\left(-\frac{35}{N_{c}+18}+\frac{56}{N_{c}+21}+\frac{99}{N_{c}+56}+\frac{35}{N_{c}+60}+\frac{21}{N_{c}+630}-\frac{176}{N_{c}}\right), \\
& \frac{1}{2520}\left(-\frac{35}{N_{c}+18}+\frac{56}{N_{c}+21}+\frac{99}{N_{c}+56}+\frac{35}{\mathrm{~N}+60}+\frac{21}{N_{c}+630}-\frac{176}{N_{c}}\right), \\
& \frac{1}{2520}\left(\frac{35}{N_{c}+18}+\frac{28}{N_{c}+21}+\frac{27}{N_{c}+56}-\frac{35}{N_{c}+60}+\frac{21}{N_{c}+630}-\frac{76}{N_{c}}\right), \\
& \left.\frac{1}{2520}\left(\frac{35}{N_{c}+18}+\frac{28}{N_{c}+21}+\frac{27}{N_{c}+56}-\frac{35}{N_{c}+60}+\frac{21}{N_{c}+630}-\frac{76}{N_{c}}\right)\right\} \\
G_{16,17,18,19,1(10)=}^{-1} & \frac{1}{2520}\left(-\frac{105}{N_{c}+18}+\frac{45}{N_{c}+56}-\frac{105}{N_{c}+60}+\frac{21}{N_{c}+630}+\frac{144}{N_{c}}\right), \\
& \frac{1}{2520}\left(-\frac{35}{N_{c}+18}+\frac{56}{N_{c}+21}+\frac{99}{N_{c}+56}+\frac{35}{N_{c}+60}+\frac{21}{N_{c}+630}-\frac{176}{N_{c}}\right), \\
& \frac{1}{2520}\left(-\frac{35}{N_{c}+18}-\frac{112}{N_{c}+21}+\frac{27}{N_{c}+56}+\frac{35}{N_{c}+60}+\frac{21}{N_{c}+630}+\frac{64}{N_{c}}\right), \\
& \frac{1}{2520}\left(\frac{35}{N_{c}+18}+\frac{28}{N_{c}+21}+\frac{27}{N_{c}+56}-\frac{35}{N_{c}+60}+\frac{21}{N_{c}+630}-\frac{76}{N_{c}}\right), \\
& \left.\frac{1}{2520}\left(\frac{35}{N_{c}+18}+\frac{28}{N_{c}+21}+\frac{27}{N_{c}+56}-\frac{35}{N_{c}+60}+\frac{21}{N_{c}+630}-\frac{76}{N_{c}}\right)\right\}
\end{aligned}
$$




$$
\begin{aligned}
& G_{1(11), 1(12), 1(13), 1(14), 1(15)=}\left\{\frac{1}{2520}\left(-\frac{35}{N_{c}+18}+\frac{56}{N_{c}+21}-\frac{81}{N_{c}+56}+\frac{35}{N_{c}+60}+\frac{21}{N_{c}+630}+\frac{4}{N_{c}}\right),\right. \\
& \frac{1}{2520}\left(\frac{35}{N_{c}+18}-\frac{56}{N_{c}+21}-\frac{9}{N_{c}+56}-\frac{35}{N_{c}+60}+\frac{21}{N_{c}+630}+\frac{44}{N_{c}}\right), \\
& \frac{1}{2520}\left(\frac{35}{N_{c}+18}+\frac{28}{N_{c}+21}+\frac{27}{N_{c}+56}-\frac{35}{N_{c}+60}+\frac{21}{N_{c}+630}-\frac{76}{N_{c}}\right), \\
& \frac{1}{2520}\left(-\frac{35}{N_{c}+18}+\frac{56}{N_{c}+21}-\frac{81}{N_{c}+56}+\frac{35}{N_{c}+60}+\frac{21}{N_{c}+630}+\frac{4}{N_{c}}\right), \\
&\left.\frac{1}{2520}\left(-\frac{105}{N_{c}+18}+\frac{45}{N_{c}+56}-\frac{105}{N_{c}+60}+\frac{21}{N_{c}+630}+\frac{144}{N_{c}}\right)\right\}
\end{aligned}
$$

$G_{1(16), 1(17), 1(18), 1(19), 1(20)}^{-1}=\left\{\frac{1}{2520}\left(\frac{35}{N_{c}+18}-\frac{56}{N_{c}+21}-\frac{9}{N_{c}+56}-\frac{35}{N_{c}+60}+\frac{21}{N_{c}+630}+\frac{44}{N_{c}}\right)\right.$,$$
\frac{1}{840}\left(\frac{35}{N_{c}+18}-\frac{56}{N_{c}+21}-\frac{9}{N_{c}+56}+\frac{35}{N_{c}+60}+\frac{7}{N_{c}+630}-\frac{12}{N_{c}}\right) \text {, }
$$$$
\frac{1}{2520}\left(-\frac{35}{N_{c}+18}-\frac{112}{N_{c}+21}+\frac{27}{N_{c}+56}+\frac{35}{N_{c}+60}+\frac{21}{N_{c}+630}+\frac{64}{N_{c}}\right) \text {, }
$$$$
\frac{1}{2520}\left(\frac{35}{N_{c}+18}+\frac{28}{N_{c}+21}+\frac{27}{N_{c}+56}-\frac{35}{N_{c}+60}+\frac{21}{N_{c}+630}-\frac{76}{N_{c}}\right) \text {, }
$$$$
\left.\frac{1}{2520}\left(\frac{35}{N_{c}+18}-\frac{56}{N_{c}+21}-\frac{9}{N_{c}+56}-\frac{35}{N_{c}+60}+\frac{21}{N_{c}+630}+\frac{44}{N_{c}}\right)\right\}
$$

$$
\begin{aligned}
G_{1(21), 1(22), 1(23), 1(24), 1(25)=}^{-1} & \frac{1}{2520}\left(\frac{35}{N_{c}+18}-\frac{56}{N_{c}+21}-\frac{9}{N_{c}+56}-\frac{35}{N_{c}+60}+\frac{21}{N_{c}+630}+\frac{44}{N_{c}}\right), \\
& \frac{1}{2520}\left(-\frac{35}{N_{c}+18}-\frac{112}{N_{c}+21}+\frac{27}{N_{c}+56}+\frac{35}{N_{c}+60}+\frac{21}{N_{c}+630}+\frac{64}{N_{c}}\right), \\
& \frac{1}{2520}\left(-\frac{35}{N_{c}+18}-\frac{112}{N_{c}+21}+\frac{27}{N_{c}+56}+\frac{35}{N_{c}+60}+\frac{21}{N_{c}+630}+\frac{64}{N_{c}}\right), \\
& \frac{1}{2520}\left(-\frac{35}{N_{c}+18}+\frac{56}{N_{c}+21}+\frac{99}{N_{c}+56}+\frac{35}{N_{c}+60}+\frac{21}{N_{c}+630}-\frac{176}{N_{c}}\right), \\
& \left.\frac{1}{2520}\left(-\frac{35}{N_{c}+18}+\frac{56}{N_{c}+21}+\frac{99}{N_{c}+56}+\frac{35}{N_{c}+60}+\frac{21}{N_{c}+630}-\frac{176}{N_{c}}\right)\right\}(3
\end{aligned}
$$

$$
\begin{aligned}
G_{1(26), 1(27), 1(28), 1(29), 1(30)=}^{-1} & \frac{1}{2520}\left(-\frac{35}{N_{c}+18}-\frac{112}{N_{c}+21}+\frac{27}{N_{c}+56}+\frac{35}{\mathrm{Nc}+60}+\frac{21}{N_{c}+630}+\frac{64}{N_{c}}\right), \\
& \frac{1}{2520}\left(-\frac{35}{N_{c}+18}-\frac{112}{N_{c}+21}+\frac{27}{N_{c}+56}+\frac{35}{N_{c}+60}+\frac{21}{N_{c}+630}+\frac{64}{N_{c}}\right), \\
& \frac{1}{2520}\left(\frac{35}{N_{c}+18}-\frac{56}{N_{c}+21}-\frac{9}{N_{c}+56}-\frac{35}{N_{c}+60}+\frac{21}{N_{c}+630}+\frac{44}{N_{c}}\right), \\
& \frac{1}{2520}\left(\frac{35}{N_{c}+18}-\frac{56}{N_{c}+21}-\frac{9}{N_{c}+56}-\frac{35}{N_{c}+60}+\frac{21}{N_{c}+630}+\frac{44}{N_{c}}\right), \\
& \left.\frac{1}{2520}\left(\frac{35}{N_{c}+18}+\frac{28}{N_{c}+21}+\frac{27}{N_{c}+56}-\frac{35}{N_{c}+60}+\frac{21}{N_{c}+630}-\frac{76}{N_{c}}\right)\right\}
\end{aligned}
$$




$$
\begin{aligned}
G_{1(31), 1(32), 1(33), 1(34), 1(35)=}^{-1} & \frac{1}{2520}\left(\frac{35}{N_{c}+18}+\frac{28}{N_{c}+21}+\frac{27}{N_{c}+56}-\frac{35}{N c+60}+\frac{21}{N_{c}+630}-\frac{76}{N_{c}}\right), \\
& \frac{1}{2520}\left(\frac{35}{N_{c}+18}-\frac{56}{N_{c}+21}-\frac{9}{N_{c}+56}-\frac{35}{N_{c}+60}+\frac{21}{N_{c}+630}+\frac{44}{N_{c}}\right), \\
& \frac{1}{2520}\left(\frac{35}{N_{c}+18}+\frac{28}{N_{c}+21}+\frac{27}{N_{c}+56}-\frac{35}{N_{c}+60}+\frac{21}{N_{c}+630}-\frac{76}{N_{c}}\right), \\
& \frac{1}{2520}\left(-\frac{35}{N_{c}+18}+\frac{56}{N_{c}+21}-\frac{81}{N_{c}+56}+\frac{35}{N_{c}+60}+\frac{21}{N_{c}+630}+\frac{4}{N_{c}}\right), \\
& \left.\frac{1}{2520}\left(-\frac{35}{N_{c}+18}+\frac{56}{N_{c}+21}-\frac{81}{N_{c}+56}+\frac{35}{N_{c}+60}+\frac{21}{N_{c}+630}+\frac{4}{N_{c}}\right)\right\}(3.4
\end{aligned}
$$

$G_{1(36), 1(37), 1(38), 1(39), 1(40)}^{-1}=\left\{\frac{1}{2520}\left(\frac{35}{N_{c}+18}+\frac{112}{N_{c}+21}-\frac{117}{N_{c}+56}-\frac{35}{N_{c}+60}+\frac{21}{N_{c}+630}-\frac{16}{N_{c}}\right)\right.$,

$$
\begin{aligned}
& \frac{1}{2520}\left(\frac{35}{N_{c}+18}-\frac{56}{N_{c}+21}-\frac{9}{N_{c}+56}-\frac{35}{N_{c}+60}+\frac{21}{N_{c}+630}+\frac{44}{N_{c}}\right), \\
& \frac{1}{2520}\left(-\frac{35}{N_{c}+18}+\frac{56}{N_{c}+21}-\frac{81}{N_{c}+56}+\frac{35}{N_{c}+60}+\frac{21}{N_{c}+630}+\frac{4}{N_{c}}\right),
\end{aligned}
$$$$
\frac{1}{2520}\left(-\frac{35}{N_{c}+18}+\frac{56}{N_{c}+21}-\frac{81}{N_{c}+56}+\frac{35}{N_{c}+60}+\frac{21}{N_{c}+630}+\frac{4}{N_{c}}\right) \text {, }
$$$$
\left.\frac{1}{2520}\left(-\frac{35}{N_{c}+18}+\frac{56}{N_{c}+21}-\frac{81}{\mathrm{Nc}+56}+\frac{35}{N_{c}+60}+\frac{21}{N_{c}+630}+\frac{4}{N_{c}}\right)\right\}
$$

$$
\begin{aligned}
G_{1(41), 1(42), 1(43), 1(44), 1(45)=}^{-1} & \frac{1}{2520}\left(\frac{35}{N_{c}+18}+\frac{112}{N_{c}+21}-\frac{117}{N_{c}+56}-\frac{35}{N_{c}+60}+\frac{21}{N_{c}+630}-\frac{16}{N_{c}}\right), \\
& \frac{1}{2520}\left(-\frac{35}{N_{c}+18}+\frac{56}{N_{c}+21}-\frac{81}{N_{c}+56}+\frac{35}{N_{c}+60}+\frac{21}{N_{c}+630}+\frac{4}{N_{c}}\right), \\
& \frac{1}{2520}\left(\frac{35}{N_{c}+18}+\frac{28}{N_{c}+21}+\frac{27}{N_{c}+56}-\frac{35}{N_{c}+60}+\frac{21}{N_{c}+630}-\frac{76}{N_{c}}\right), \\
& \frac{1}{2520}\left(\frac{35}{N_{c}+18}-\frac{56}{N_{c}+21}-\frac{9}{N_{c}+56}-\frac{35}{N_{c}+60}+\frac{21}{N_{c}+630}+\frac{44}{N_{c}}\right), \\
& \left.\frac{1}{2520}\left(-\frac{35}{N_{c}+18}+\frac{56}{N_{c}+21}-\frac{81}{N_{c}+56}+\frac{35}{N_{c}+60}+\frac{21}{N_{c}+630}+\frac{4}{N_{c}}\right)\right\}
\end{aligned}
$$

$$
\begin{aligned}
& G_{1(46), 1(47), 1(48), 1(49), 1(50)=}\left\{\frac{1}{2520}\left(\frac{35}{N_{c}+18}+\frac{112}{N_{c}+21}-\frac{117}{N_{c}+56}-\frac{35}{N_{c}+60}+\frac{21}{N_{c}+630}-\frac{16}{N_{c}}\right),\right. \\
& \frac{1}{2520}\left(-\frac{105}{N_{c}+18}+\frac{45}{N_{c}+56}-\frac{105}{N_{c}+60}+\frac{21}{N_{c}+630}+\frac{144}{N_{c}}\right), \\
& \frac{1}{2520}\left(\frac{35}{N_{c}+18}+\frac{28}{N_{c}+21}+\frac{27}{N_{c}+56}-\frac{35}{N_{c}+60}+\frac{21}{N_{c}+630}-\frac{76}{N_{c}}\right), \\
& \frac{1}{2520}\left(\frac{35}{N_{c}+18}-\frac{56}{N_{c}+21}-\frac{9}{N_{c}+56}-\frac{35}{N_{c}+60}+\frac{21}{N_{c}+630}+\frac{44}{N_{c}}\right), \\
&\left.\frac{1}{2520}\left(-\frac{35}{N_{c}+18}+\frac{56}{N_{c}+21}-\frac{81}{N_{c}+56}+\frac{35}{N_{c}+60}+\frac{21}{N_{c}+630}+\frac{4}{N_{c}}\right)\right\}
\end{aligned}
$$




$$
\begin{aligned}
& G_{1(51), 1(52), 1(53), 1(54), 1(55)}^{-1}=\left\{\frac{1}{840}\left(\frac{35}{N_{c}+18}-\frac{56}{N_{c}+21}-\frac{9}{N_{c}+56}+\frac{35}{N_{c}+60}+\frac{7}{N_{c}+630}-\frac{12}{N_{c}}\right),\right. \\
& \frac{1}{2520}\left(-\frac{35}{N_{c}+18}-\frac{112}{\mathrm{Nc}+21}+\frac{27}{N_{c}+56}+\frac{35}{N_{c}+60}+\frac{21}{N_{c}+630}+\frac{64}{\mathrm{Nc}}\right) \text {, } \\
& \frac{1}{2520}\left(-\frac{35}{N_{c}+18}+\frac{56}{N_{c}+21}-\frac{81}{N_{c}+56}+\frac{35}{\operatorname{textNc}+60}+\frac{21}{N_{c}+630}+\frac{4}{N_{c}}\right) \text {, } \\
& \frac{1}{2520}\left(-\frac{35}{N_{c}+18}+\frac{56}{N_{c}+21}-\frac{81}{N_{c}+56}+\frac{35}{N_{c}+60}+\frac{21}{N_{c}+630}+\frac{4}{N_{c}}\right) \text {, } \\
& \left.\frac{1}{2520}\left(\frac{35}{N_{c}+18}+\frac{28}{N_{c}+21}+\frac{27}{N_{c}+56}-\frac{35}{N_{c}+60}+\frac{21}{N_{c}+630}-\frac{76}{N_{c}}\right)\right\} \\
& G_{1(56), 1(57), 1(58), 1(59), 1(60)}^{-1}=\left\{\frac{1}{2520}\left(\frac{35}{N_{c}+18}-\frac{56}{N_{c}+21}-\frac{9}{N_{c}+56}-\frac{35}{N_{c}+60}+\frac{21}{N_{c}+630}+\frac{44}{N_{c}}\right)\right. \\
& \frac{1}{2520}\left(\frac{35}{N_{c}+18}-\frac{56}{N_{c}+21}-\frac{9}{N_{c}+56}-\frac{35}{N_{c}+60}+\frac{21}{N_{c}+630}+\frac{44}{N_{c}}\right) \text {, } \\
& \frac{1}{2520}\left(-\frac{35}{N_{c}+18}-\frac{112}{N_{c}+21}+\frac{27}{N_{c}+56}+\frac{35}{N_{c}+60}+\frac{21}{N_{c}+630}+\frac{64}{N_{c}}\right) \text {, } \\
& \frac{1}{2520}\left(-\frac{35}{N_{c}+18}-\frac{112}{N_{c}+21}+\frac{27}{N_{c}+56}+\frac{35}{N_{c}+60}+\frac{21}{N_{c}+630}+\frac{64}{N_{c}}\right) \text {, } \\
& \left.\frac{1}{2520}\left(-\frac{35}{N_{c}+18}+\frac{56}{N_{c}+21}+\frac{99}{N_{c}+56}+\frac{35}{N_{c}+60}+\frac{21}{N_{c}+630}-\frac{176}{N_{c}}\right)\right\}
\end{aligned}
$$

Remarks. Before concluding this section, let us make a few remarks on the degrees of freedom introduced by $N_{c}$. First we notice that $\operatorname{det}(G)$ will depend on $N_{c}$, thus there are solutions of $N_{c}$ such that $\operatorname{det}(G)=0$. When this happens, $G \cdot \tau=n$ will not have solution. In other words, for these specific values, $N_{c}$ and the imposed loop-KK relations are not compatible to each other. At this moment, we are not clear what is the physical meaning of these degenerated values of $N_{c}$. However from explicit examples discussed above, it seems that $N_{c}$ that lead to degenerating matrix $G$ are always negative integer. For positive $N_{c}$ there is no problem for it. It is perhaps possible to choose special values of $N_{c}$ such that the final expression dramatically simplifies or manifest patten can be observed.

\section{An alternative approach}

In previous section, we solved $\tau$ by $n$ using the $G$-matrix directly. Since all conditions we imposed, such as (3.9), (3.10), (3.11) and (3.12), are relabeling symmetric, the solutions $\tau_{\sigma}$ for different ordering $\sigma$ 's are also related by relabeling symmetry. This property can be used to solve $\tau$ without using the $G$-matrix, which will be the purpose of this section. In fact, similar method has been used in tree-level case in [40]. For simplicity, in this section we assume $N_{c}=1$. 
Four-point example. In the four-point case we assume that $\tau$ can be expanded by $n$, i.e.,

$$
\tau_{1234}=a n_{1234}+b n_{1243}+c n_{1324}
$$

Under the relabeling $1 \leftrightarrow 2$, we get

$$
\tau_{2134}=a n_{2134}+b n_{2143}+c n_{2314},
$$

which can be recast into the original basis using reflection and cyclic symmetry of $\tau$ and $n$

$$
\tau_{1342}=a n_{1243}+b n_{1234}+c n_{1324} .
$$

Same $\tau_{1342}$ can also obtained from $\tau_{1234}$ by relabeling $2 \rightarrow 3,3 \rightarrow 4,4 \rightarrow 2$, thus we arrive following equation

$$
\tau_{1342}=a n_{1342}+b n_{1324}+c n_{1432}=a n_{1243}+b n_{1324}+c n_{1234} .
$$

By comparing the $\tau_{1342}$ in this two different ways, we can get

$$
b=c .
$$

Thus

$$
\tau_{a b c d}=a n_{a b c d}+b\left(n_{a b d c}+n_{a c b d}\right) .
$$

Substituting this into the relation between $n$ and $\tau$ (3.16), we get

$$
a=\frac{21}{62}, \quad b=-\frac{5}{31} .
$$

Then

$$
\tau_{a b c d}=\frac{21}{62} n_{a b c d}-\frac{5}{31}\left(n_{a b d c}+n_{a c b d}\right) .
$$

This is the same with the result obtained by imposing KK relation and then solving linear equations.

Five-point expansion. Similarly at five-points, we assume the dual trace can be expanded into the $(5-1) ! / 2=12$ basis numerators $n_{1, \sigma}$ discussed in section 3.3,

$$
\begin{aligned}
\tau_{12345} & =\sum_{\sigma \in S_{4} / R} c_{1, \sigma} n_{1, \sigma} \\
& =c_{12345} n_{12345}+c_{12354} n_{12354}+\cdots+c_{14325} n_{14325},
\end{aligned}
$$

where $R$ denotes reflection. Comparing the expansion expressions derived through permutating leg 1 with 2, 3, 4, 5 with the corresponding expressions obtained by relabeling, we 
get the following relations

$$
\begin{aligned}
& \tau_{21345}=-\tau_{12543} \longrightarrow c_{12453}=c_{12534}, \quad c_{12354}=c_{12435}, \\
& c_{13254}=-c_{13425}, c_{13245}=-c_{14325}, \\
& \tau_{32145}=-\tau_{12354} \longrightarrow c_{12534}=c_{14235}, \quad c_{12543}=-c_{13245}, \\
& c_{12435}=-c_{14325}, c_{12453}=-c_{13254} \text {, } \\
& \tau_{42315}=-\tau_{13245} \longrightarrow c_{13254}=-c_{14235}, c_{12453}=c_{13425} \\
& c_{12435}=-c_{12543}, c_{12354}=-c_{14325}, \\
& \tau_{52341}=-\tau_{14325} \longrightarrow c_{12534}=-c_{13254}, c_{13425}=c_{14235}, \\
& c_{12354}=-c_{12543}, c_{12435}=c_{13245} .
\end{aligned}
$$

Relabling symmetry therefore reduces the number of independent coefficients to four, yielding

$$
\begin{aligned}
\tau_{12345}= & a n_{12345}++b\left(n_{12453}+n_{12534}-n_{13254}+n_{13425}+n_{14235}\right) \\
& c n_{13524}-d\left(n_{12354}+n_{12435}-n_{12543}+n_{13245}-n_{14325}\right),
\end{aligned}
$$

while the other basis dual traces $\tau$ s can be obtained by relabelings of legs $2,3,4$ and 5 . Substituting these expressions back to just one relation (3.28) allows us to fully determine the remaining all four coefficients. Again, we arrive at

$$
\begin{aligned}
\tau_{\{12345\}}= & \frac{1}{62}\left(16 n_{12345}-6 n_{12354}-6 n_{12435}-3 n_{12453}-3 n_{12534}+6 n_{12543}\right. \\
& \left.-6 n_{13245}+3 n_{13254}-3 n_{13425}-3 n_{14235}+6 n_{14325}\right) .
\end{aligned}
$$

\section{Conclusion}

In this paper we have discussed two kinds of dual-color decompositions of Yang-Mills amplitude at one-loop level. Starting from an existing double-copy formulation we showed that the Yang-Mills integrand can be translated into dual DDM form up to terms that vanish upon integration. Once a dual DDM form is obtained, we showed that it is possible to take one step further and write down the corresponding dual trace-form. In particular we saw that the similar technique used to determine tree-level dual trace generalizes to one-loop. In a sense that the additional degrees of freedom introduced by loop momentum shifting which potentially may cause problem has been taken care of during the translation between BCJ and dual DDM form.

Without the knowledge of an actual kinematic algebra as input the algorithm presented in this paper uses Kleiss-Kuijf relation and reflection conditions as substitutes and we discussed the possibility of generically solving dual traces in terms of one-loop numerators. We expect that there might be an algebraic realization of the dual trace analogous to what has discussed at tree-level in [41], or more interestingly, if the algebraic structure observed in the scattering equations approach [19] could be systematically generalized to loop-level. Note especially that although in this paper we constructed the dual traces 
assuming BCJ numerators were determined in advance, this operation can be reversed. The relation between $\tau$ and $n_{i}$ provides an alternative derivation of the numerators if the $\tau$ s can be determined from an independent method, for example from the recent work of Naculich [43], or from the method outlined by the current authors in [44].

\section{Acknowledgments}

Y.J. Du would like to acknowledge the EU programme Erasmus Mundus Action 2 and the International Postdoctoral Exchange Fellowship Program of China for supporting his postdoctoral research in Lund University(with Fudan University as the home university). Y.J. Du's research is supported in parts by the NSF of China Grant No.11105118, China Postdoctoral Science Foundation No.2013M530175 and the Fundamental Research Funds for the Central Universities of Fudan University No.20520133169. C.F. would like to acknowledge the support from National Science Council, 50 billions project of Ministry of Education and National Center for Theoretical Science, Taiwan, Republic of China as well as the support from S.T. Yau center of National Chiao Tung University. B.F is supported, in part, by fund from Qiu-Shi and Chinese NSF funding under contract No.11031005, No.11135006, No.11125523.

Open Access. This article is distributed under the terms of the Creative Commons Attribution License (CC-BY 4.0), which permits any use, distribution and reproduction in any medium, provided the original author(s) and source are credited.

\section{References}

[1] Z. Bern, J.J.M. Carrasco and H. Johansson, New Relations for Gauge-Theory Amplitudes, Phys. Rev. D 78 (2008) 085011 [arXiv:0805.3993] [INSPIRE].

[2] N.E.J. Bjerrum-Bohr, P.H. Damgaard and P. Vanhove, Minimal Basis for Gauge Theory Amplitudes, Phys. Rev. Lett. 103 (2009) 161602 [arXiv:0907.1425] [INSPIRE].

[3] S. Stieberger, Open \& Closed vs. Pure Open String Disk Amplitudes, arXiv:0907.2211 [INSPIRE].

[4] F. Cachazo, Fundamental BCJ Relation in $N=4$ SYM From The Connected Formulation, arXiv: 1206.5970 [INSPIRE].

[5] F. Cachazo, S. He and E.Y. Yuan, Scattering Equations and KLT Orthogonality, arXiv:1306.6575 [INSPIRE].

[6] F. Cachazo, S. He and E.Y. Yuan, Scattering of Massless Particles in Arbitrary Dimension, arXiv:1307.2199 [INSPIRE].

[7] F. Cachazo, S. He and E.Y. Yuan, Scattering of Massless Particles: Scalars, Gluons and Gravitons, arXiv:1309.0885 [INSPIRE].

[8] B. Feng, R. Huang and Y. Jia, Gauge Amplitude Identities by On-shell Recursion Relation in S-matrix Program, Phys. Lett. B 695 (2011) 350 [arXiv: 1004.3417] [INSPIRE].

[9] Y. Jia, R. Huang and C.-Y. Liu, U(1)-decoupling, KK and BCJ relations in $\mathcal{N}=4 S Y M$, Phys. Rev. D 82 (2010) 065001 [arXiv: 1005.1821] [INSPIRE]. 
[10] Y.-X. Chen, Y.-J. Du and B. Feng, A proof of the explicit minimal-basis expansion of tree amplitudes in gauge field theory, JHEP 02 (2011) 112 [arXiv:1101.0009] [INSPIRE].

[11] T. Sondergaard, New Relations for Gauge-Theory Amplitudes with Matter, Nucl. Phys. B 821 (2009) 417 [arXiv:0903.5453] [INSPIRE].

[12] S.H. Henry Tye and Y. Zhang, Dual Identities inside the Gluon and the Graviton Scattering Amplitudes, JHEP 06 (2010) 071 [Erratum ibid. 1104 (2011) 114] [arXiv:1003.1732] [INSPIRE].

[13] N.E.J. Bjerrum-Bohr, P.H. Damgaard, T. Sondergaard and P. Vanhove, Monodromy and Jacobi-like Relations for Color-Ordered Amplitudes, JHEP 06 (2010) 003 [arXiv:1003.2403] [INSPIRE].

[14] H. Tye and Y. Zhang, Remarks on the identities of gluon tree amplitudes, Phys. Rev. D 82 (2010) 087702 [arXiv: 1007.0597] [INSPIRE].

[15] C.R. Mafra, O. Schlotterer and S. Stieberger, Explicit BCJ numerators from pure spinors, JHEP 07 (2011) 092 [arXiv: 1104.5224] [INSPIRE].

[16] R. Monteiro and D. O'Connell, The kinematic algebra from the self-dual sector, JHEP 07 (2011) 007 [arXiv: 1105.2565] [INSPIRE].

[17] N.E.J. Bjerrum-Bohr, P.H. Damgaard, R. Monteiro and D. O'Connell, Algebras for amplitudes, JHEP 06 (2012) 061 [arXiv: 1203.0944] [INSPIRE].

[18] C.-H. Fu, Y.-J. Du and B. Feng, An algebraic approach to BCJ numerators, JHEP 03 (2013) 050 [arXiv: 1212.6168] [INSPIRE].

[19] R. Monteiro and D. O'Connell, The kinematic algebras from the scattering equations, JHEP 03 (2014) 110 [arXiv:1311.1151] [INSPIRE].

[20] T. Sondergaard, Perturbative Gravity and Gauge Theory Relations: A Review, Adv. High Energy Phys. 2012 (2012) 726030 [arXiv:1106.0033] [InSPIRE].

[21] N.E.J. Bjerrum-Bohr, P.H. Damgaard, H. Johansson and T. Sondergaard, Monodromy-like relations for finite loop amplitudes, JHEP 05 (2011) 039 [arXiv:1103.6190] [INSPIRE].

[22] R.H. Boels and R.S. Isermann, New relations for scattering amplitudes in Yang-Mills theory at loop level, Phys. Rev. D 85 (2012) 021701 [arXiv:1109.5888] [InSPIRE].

[23] R.H. Boels and R.S. Isermann, Yang-Mills amplitude relations at loop level from non-adjacent BCFW shifts, JHEP 03 (2012) 051 [arXiv:1110.4462] [INSPIRE].

[24] Y.-J. Du and H. Lüo, On General BCJ Relation at One-loop Level in Yang-Mills Theory, JHEP 01 (2013) 129 [arXiv:1207.4549] [INSPIRE].

[25] R.H. Boels, B.A. Kniehl, O.V. Tarasov and G. Yang, Color-kinematic Duality for Form Factors, JHEP 02 (2013) 063 [arXiv: 1211.7028] [INSPIRE].

[26] J.J.M. Carrasco, M. Chiodaroli, M. Günaydin and R. Roiban, One-loop four-point amplitudes in pure and matter-coupled $N \leq 4$ supergravity, JHEP 03 (2013) 056 [arXiv: 1212.1146] [INSPIRE].

[27] J.J. Carrasco and H. Johansson, Five-Point Amplitudes in $N=4$ super-Yang-Mills Theory and $N=8$ Supergravity, Phys. Rev. D 85 (2012) 025006 [arXiv:1106.4711] [INSPIRE].

[28] N.E.J. Bjerrum-Bohr, T. Dennen, R. Monteiro and D. O'Connell, Integrand Oxidation and One-Loop Colour-Dual Numerators in N=4 Gauge Theory, JHEP 07 (2013) 092 [arXiv: 1303.2913] [INSPIRE]. 
[29] Z. Bern, S. Davies, T. Dennen, Y.-t. Huang and J. Nohle, Color-Kinematics Duality for Pure Yang-Mills and Gravity at One and Two Loops, arXiv:1303.6605 [INSPIRE].

[30] R.H. Boels, R.S. Isermann, R. Monteiro and D. O'Connell, Colour-Kinematics Duality for One-Loop Rational Amplitudes, JHEP 04 (2013) 107 [arXiv: 1301.4165] [INSPIRE].

[31] J. Nohle, Color-Kinematics Duality in One-Loop Four-Gluon Amplitudes with Matter, arXiv:1309.7416 [INSPIRE].

[32] R. Kleiss and H. Kuijf, Multi-Gluon Cross-sections and Five Jet Production at Hadron Colliders, Nucl. Phys. B 312 (1989) 616 [inSPIRE].

[33] V. Del Duca, L.J. Dixon and F. Maltoni, New color decompositions for gauge amplitudes at tree and loop level, Nucl. Phys. B 571 (2000) 51 [hep-ph/9910563] [INSPIRE].

[34] H. Kawai, D.C. Lewellen and S.H.H. Tye, A Relation Between Tree Amplitudes of Closed and Open Strings, Nucl. Phys. B 269 (1986) 1 [InSPIRE].

[35] Y.-J. Du, B. Feng and C.-H. Fu, BCJ Relation of Color Scalar Theory and KLT Relation of Gauge Theory, JHEP 08 (2011) 129 [arXiv:1105.3503] [inSPIRE].

[36] Z. Bern, A. De Freitas and H.L. Wong, On the coupling of gravitons to matter, Phys. Rev. Lett. 84 (2000) 3531 [hep-th/9912033] [INSPIRE].

[37] Z. Bern, T. Dennen, Y.-t. Huang and M. Kiermaier, Gravity as the Square of Gauge Theory, Phys. Rev. D 82 (2010) 065003 [arXiv: 1004.0693] [INSPIRE].

[38] Z. Bern and T. Dennen, A Color Dual Form for Gauge-Theory Amplitudes, Phys. Rev. Lett. 107 (2011) 081601 [arXiv:1103.0312] [INSPIRE].

[39] Z. Bern, C. Boucher-Veronneau and H. Johansson, $N \geq 4$ Supergravity Amplitudes from Gauge Theory at One Loop, Phys. Rev. D 84 (2011) 105035 [arXiv:1107.1935] [INSPIRE].

[40] Y.-J. Du, B. Feng and C.-H. Fu, The Construction of Dual-trace Factor in Yang-Mills Theory, JHEP 07 (2013) 057 [arXiv: 1304 . 2978] [INSPIRE].

[41] C.-H. Fu, Y.-J. Du and B. Feng, Note on Construction of Dual-trace Factor in Yang-Mills Theory, JHEP 10 (2013) 069 [arXiv: 1305.2996] [INSPIRE].

[42] Z. Bern, L.J. Dixon, D.C. Dunbar and D.A. Kosower, One loop $n$ point gauge theory amplitudes, unitarity and collinear limits, Nucl. Phys. B 425 (1994) 217 [hep-ph/9403226] [INSPIRE].

[43] S.G. Naculich, Scattering equations and virtuous kinematic numerators and dual-trace functions, arXiv:1404.7141 [INSPIRE].

[44] C.-H. Fu, Y.-J. Du and B. Feng, Note on symmetric BCJ numerator, journal (2014) [arXiv:1403.6262] [INSPIRE]. 